\title{
High scale impact in alignment and decoupling in two-Higgs-doublet models
}

\author{
Philipp Basler, ${ }^{1, *}$ Pedro M. Ferreira, ${ }^{2,3, \dagger}$ Margarete Mühlleitner, ${ }^{1, *}$ and Rui Santos ${ }^{2,3,4, \S}$ \\ ${ }^{1}$ Institute for Theoretical Physics, Karlsruhe Institute of Technology, Karlsruhe 76128, Germany \\ ${ }^{2}$ ISEL_Instituto Superior de Engenharia de Lisboa, Instituto Politécnico de Lisboa, \\ Lisboa 1959-007, Portugal \\ ${ }^{3}$ Centro de Física Teórica e Computacional, Faculdade de Ciências, Universidade de Lisboa, \\ Campo Grande, Edifício C8, Lisboa 1749-016, Portugal \\ ${ }^{4}$ LIP, Departamento de Física, Universidade do Minho, Braga 4710-057, Portugal
}

(Received 23 November 2017; revised manuscript received 6 April 2018; published 18 May 2018)

\begin{abstract}
The two-Higgs-doublet model (2HDM) provides an excellent benchmark to study physics beyond the Standard Model (SM). In this work, we discuss how the behavior of the model at high-energy scales causes it to have a scalar with properties very similar to those of the SM-which means the 2HDM can be seen to naturally favor a decoupling or alignment limit. For a type II $2 \mathrm{HDM}$, we show that requiring the model to be theoretically valid up to a scale of $1 \mathrm{TeV}$, by studying the renormalization group equations (RGE) of the parameters of the model, causes a significant reduction in the allowed magnitude of the quartic couplings. This, combined with $B$-physics bounds, forces the model to be naturally decoupled. As a consequence, any nondecoupling limits in type II, like the wrong-sign scenario, are excluded. On the contrary, even with the very constraining limits for the Higgs couplings from the LHC, the type I model can deviate substantially from alignment. An RGE analysis similar to that made for type II shows, however, that requiring a single scalar to be heavier than about $500 \mathrm{GeV}$ would be sufficient for the model to be decoupled. Finally, we show that the $2 \mathrm{HDM}$ is stable up to the Planck scale independently of which of the $C P$-even scalars is the discovered $125 \mathrm{GeV}$ Higgs boson.
\end{abstract}

DOI: 10.1103/PhysRevD.97.095024

\section{INTRODUCTION}

The discovery of the Higgs boson at the Large Hadron Collider (LHC) by the ATLAS [1] and CMS [2] collaborations has immediately triggered the discussion about which extensions of the Standard Model (SM) could accommodate all data and still predict new physics, observable during the Run 2 operation. One of the models that has been used as benchmark for the searches for new physics by both ATLAS and CMS is the two-Higgs-doublet model (2HDM) in its $C P$-conserving, softly broken $\mathbb{Z}_{2}$ symmetric version. First proposed by T.D. Lee [3], 2HDMs have been used as benchmark models not only for the LHC searches but also theoretically. The different versions of the model allow for instance the introduction of $C P$ violation in the scalar sector, controlled flavor-changing neutral

\footnotetext{
"philipp.basler@kit.edu

pmmferreira@fc.ul.pt

*margarete.muehlleitner@kit.edu

rasantos@fc.ul.pt
}

Published by the American Physical Society under the terms of the Creative Commons Attribution 4.0 International license. Further distribution of this work must maintain attribution to the author(s) and the published article's title, journal citation, and DOI. Funded by SCOAP ${ }^{3}$. currents or dark matter candidates. The models also have a very different vacuum structure than the SM one, because both charge and $C P$ can be broken spontaneously. Furthermore, it is the simplest extension where simultaneous minima of the same nature can occur.

With the mass of the Higgs boson determined with a good precision, the discussion about the stability of the SM Higgs potential has restarted. This involves studying the evolution of the SM quartic coupling $\lambda$ with the renormalization group equations (RGE). Two effects are here in play: on the one hand, the quartic coupling itself has a positive contribution to its own RGE, and therefore tends to increase its value as one goes to higher energy scales; on the other hand, the top quark Yukawa coupling has a negative contribution to the RGE of $\lambda$, and tends to reduce its value as one goes up in energy scale. As a result of these two effects, if the value of the quartic coupling at the weak scale is too small, the RGE evolution will cause $\lambda$ to turn negative at some point, and therefore the potential becomes unstable. If, however, the starting value of the quartic coupling is too large, its RGE evolution will drive it to ever-higher values so that the theory ceases eventually to be perturbative and $\lambda$ develops a Landau pole. Prior to the Higgs boson discovery, these arguments were used to constrain its mass [4-13]. Now that we know its mass, we can verify whether the 
potential remains stable, and the theory perturbative, all the way up to the Planck scale. If that were not the case, that would most likely be a sign of the existence of new physics, hitherto undiscovered, which would stabilize the RGE evolution of the couplings. It has been shown, in fact, that the SM vacuum is metastable if the theory is to be valid up to the Planck scale [14-16]. The only way to have a stable electroweak vacuum, according to these results, would therefore be for new physics to exist at a scale well below the Planck scale. The stability of the electroweak vacuum can be cured with the addition of extra scalar degrees of freedom. With all the parameters of the SM determined, the addition of a scalar singlet is enough to cure the problem [17-20]. As shown in [20], the addition of a complex singlet provides a vacuum stable up to the Planck scale. In the broken phase of the model, only one scalar with a mass above $125 \mathrm{GeV}$ is needed to stabilize the vacuum. Therefore, since there are two extra $C P$-even scalars, the remaining one can have a mass below $125 \mathrm{GeV}$. It has been shown, however, in the context of the SM, that the presence of new physics very close to the Planck scale can alter considerably such conditions of stability of the potential [21-23], and likewise eventual gravity contributions near the Planck scale can have a sizeable impact [24].

The 2HDM belongs to the simplest extensions of the SM. An extra scalar doublet enlarges the SM scalar model, but the remaining fields (gauge and fermion) remain the same, as do the gauge symmetries of the model. A larger scalar sector implies a more complex scalar potentialindeed the version of the 2HDM potential in this work has 5 quartic couplings. And as in the SM, one can ask whether the potential remains stable and perturbative, as one considers progressively larger energy scales. As such, the RGE evolutions of the quartic couplings of the 2HDM were studied by several authors [25-28] to ascertain the validity of the model up to higher energy scales and, prior to the Higgs discovery, to attempt to impose constraints on the unknown scalar masses of the model. After the Higgs boson was discovered the stability of the several versions of the 2HDM was revisited in a number of papers [29-37]. In all these works, the lightest $C P$-even scalar is considered to be the discovered Higgs boson, and there is a common conclusion that, with all relevant theoretical and experimental constraints taken into account, there always exists a region of the parameter space where the $2 \mathrm{HDM}$ is valid up to the Planck scale. Notice, however, that these studies assume a softly broken $\mathbb{Z}_{2}$ symmetry, the most popular version of the $2 \mathrm{HDM}$, and the region of parameter space found always included $m_{12}^{2} \neq 0$. On the other hand, in Ref. [29] a type II version with an exact $\mathbb{Z}_{2}$ symmetric model was analyzed, concluding that the potential with $m_{12}^{2}=0$ cannot be valid beyond $10 \mathrm{TeV}$ without the intervention of new physics, a conclusion later confirmed in [31]. However, this conclusion is heavily dependent on the value of the charged Higgs mass, $m_{H^{ \pm}}$. We will show that the type II model with an exact symmetry is, when taking into account the most recent bounds on $m_{H^{ \pm}}$, in fact valid only up to a few hundreds of GeV.

All studies quoted above agree on the fact that the quartic parameters of the potential are increasingly small if the theory is to be valid up to higher and higher scales. The issue of metastability in the 2HDM at high scales was discussed in [34] while in Ref. [36] it was shown that the heavy states of a $2 \mathrm{HDM}$ valid up to Planck scale can be probed with a significance of at least $3 \sigma$ in the LHC high-luminosity run. In this investigation we will not work in the exact alignment limit nor in the decoupling limit as was done in previous works. Our main goal will in effect be to show how 2HDM alignment may emerge "naturally" from requiring stability and perturbativity of the potential up to high-energy scales. We will furthermore scan over the entire parameter space allowed by the experimental constraints to be described later. Moreover, we will take into account the combination of all theoretical constraints, including the discriminant that forces the minimum to be global ${ }^{1}$ at various scales. An interesting conclusion we will reach is that it is enough to have only one heavy scalar boson to have decoupling (and therefore alignment) at a scale as low as $1 \mathrm{TeV}$. We will argue that decoupling can be defined for masses as low as $500 \mathrm{GeV}$-in other words, if even only one of the extra scalar masses is required to be above $500 \mathrm{GeV}$, the 2HDM with good high-energy scale behavior up to scales of the $\mathrm{TeV}$ order automatically has a scalar state with SM-like properties.

We will analyze for the first time the stability of a softly broken $\mathbb{Z}_{2}$ symmetric $2 \mathrm{HDM}$ in the case where the heaviest $C P$-even scalar is the $125 \mathrm{GeV}$ Higgs boson-the so-called heavy Higgs scenario. Quite surprisingly, we will demonstrate that for this scenario there are regions of the parameter space for a type I model for which the theory is well behaved all the way up to the Planck scale. This is only possible if all the remaining scalar bosons have a mass below $200 \mathrm{GeV}$. Obviously, due to the bound of $580 \mathrm{GeV}$ on the charged Higgs mass for a type II model, the heavy Higgs scenario in type II ceases to be valid already below an energy scale of about $1 \mathrm{TeV}$.

The paper is organized as follows. In Sec. II, we describe the version of the 2HDM used in this work, and in Sec. III, we present the theoretical constraints that we will impose at the various scales. In Sec. IV, we discuss the parameter space of the model in view of the most relevant theoretical and up to date experimental constraints. In Secs. V-VII, we present our results. Our conclusions are given in Sec. IX. In Appendix A, we have collected the relevant RGEs for this study.

\footnotetext{
${ }^{1}$ One-loop studies of the vacuum of some versions of the $2 \mathrm{HDM}$ were performed in $[38,39]$.
} 


\section{THE TWO-HIGGS-DOUBLET MODEL}

The 2HDM is an extension of the SM in which the scalar potential is built with two hypercharge 1 complex $S U(2)$ doublets $\Phi_{1}$ and $\Phi_{2}$. When all the fields transform just as in the SM and no extra symmetries are imposed on the Lagrangian, the most general Yukawa Lagrangian gives rise to tree-level flavor-changing neutral currents (FCNC) which are known to be severely constrained by experimental data. Imposing a discrete symmetry on the scalar fields, $\Phi_{1} \rightarrow \Phi_{1}, \Phi_{2} \rightarrow-\Phi_{2}$, and forcing the potential to be invariant under this $\mathbb{Z}_{2}$ symmetry, except for a dimension two soft breaking term, the potential can be written as

$$
\begin{aligned}
V\left(\Phi_{1}, \Phi_{2}\right)= & m_{11}^{2} \Phi_{1}^{\dagger} \Phi_{1}+m_{22}^{2} \Phi_{2}^{\dagger} \Phi_{2}-\left(m_{12}^{2} \Phi_{1}^{\dagger} \Phi_{2}+\text { H.c. }\right) \\
& +\frac{1}{2} \lambda_{1}\left(\Phi_{1}^{\dagger} \Phi_{1}\right)^{2}+\frac{1}{2} \lambda_{2}\left(\Phi_{2}^{\dagger} \Phi_{2}\right)^{2} \\
& +\lambda_{3}\left(\Phi_{1}^{\dagger} \Phi_{1}\right)\left(\Phi_{2}^{\dagger} \Phi_{2}\right)+\lambda_{4}\left(\Phi_{1}^{\dagger} \Phi_{2}\right)\left(\Phi_{2}^{\dagger} \Phi_{1}\right) \\
& +\frac{1}{2} \lambda_{5}\left[\left(\Phi_{1}^{\dagger} \Phi_{2}\right)^{2}+\text { H.c. }\right] .
\end{aligned}
$$

We will work with a $C P$-conserving potential by considering all parameters of the potential, together with the vacuum expectation values, to be real. Also the parameter space we will consider is such that no spontaneous $C P$ breaking occurs. This is in fact assured by simply requiring that a $C P$-preserving minimum exists [40]. When the symmetry is extended to the fermions in such a way that a fermion of a given charge couples only to one doublet $[41,42]$ the Higgs interaction terms become proportional to the quark mass terms and therefore Higgs FCNC are absent at tree level. There are four independent choices for the Yukawa Lagrangian [43]. We will call type I the model where only $\Phi_{2}$ couples to all fermions, type II the model where $\Phi_{2}$ couples to up-type quarks and $\Phi_{1}$ couples to down-type quarks and leptons, Flipped (F) the model where $\Phi_{2}$ couples to up-type quarks and to leptons and $\Phi_{1}$ couples to down-type quarks and finally Lepton Specific (LS) the model where $\Phi_{2}$ couples to all quarks and $\Phi_{1}$ couples to leptons. In this work we will focus on type I and type II as the results for type F and LS are similar to the ones for type II and type I, respectively. In fact, as we will discuss later, the major difference between the models is related to the bound on the charged Higgs mass, which is similar for type I and LS and for type II and F.

The two complex doublet fields $\Phi_{1}$ and $\Phi_{2}$ are expressed in terms of charged complex fields $\phi_{i}^{+}$and real and imaginary components of the neutral components of the doublets, $\rho_{i}$ and $\eta_{i}(i=1,2)$, respectively. After electroweak symmetry breaking (EWSB) we can expand the two doublets about their vacuum expectation values (VEVs) $v_{1}$ and $v_{2}$ yielding

$$
\Phi_{1}=\left(\begin{array}{c}
\phi_{1}^{+} \\
\frac{\rho_{1}+i \eta_{1}+v_{1}}{\sqrt{2}}
\end{array}\right) \quad \text { and } \quad \Phi_{2}=\left(\begin{array}{c}
\phi_{2}^{+} \\
\frac{\rho_{2}+i \eta_{2}+v_{2}}{\sqrt{2}}
\end{array}\right)
$$

The mass matrices are the components of the bilinear terms in the potential. As we assume charge and $C P$ conservation we end up with three $2 \times 2$ matrices $\mathcal{M}_{S}, \mathcal{M}_{P}$ and $\mathcal{M}_{C}$ for the neutral $C P$-even, neutral $C P$-odd and charged Higgs sectors. The minimization conditions are given by

$$
\left.\frac{\partial V}{\partial \Phi_{1}}\right|_{\left\langle\Phi_{i}\right\rangle}=\left.\frac{\partial V}{\partial \Phi_{2}}\right|_{\left\langle\Phi_{i}\right\rangle}=0
$$

which is equivalent to setting the two terms in the potential linear in $\rho_{1}$ and $\rho_{2}$ to zero,

$$
\begin{aligned}
& m_{11}^{2}-m_{12}^{2} \frac{v_{2}}{v_{1}}+\frac{\lambda_{1} v_{1}^{2}}{2}+\frac{\lambda_{345} v_{2}^{2}}{2}=0 \\
& m_{22}^{2}-m_{12}^{2} \frac{v_{1}}{v_{2}}+\frac{\lambda_{2} v_{2}^{2}}{2}+\frac{\lambda_{345} v_{1}^{2}}{2}=0,
\end{aligned}
$$

where we have defined

$$
\lambda_{345} \equiv \lambda_{3}+\lambda_{4}+\lambda_{5}
$$

These equations allow one to replace the $m_{11}^{2}$ and $m_{22}^{2}$ parameters by expressions in terms of the remaining parameters and the VEVs to obtain the following form for the mass matrices,

$$
\begin{aligned}
& \mathcal{M}_{S}=\left(\begin{array}{cc}
m_{12}^{2} \frac{v_{2}}{v_{1}}+\lambda_{1} v_{1}^{2} & -m_{12}^{2}+\lambda_{345} v_{1} v_{2} \\
-m_{12}^{2}+\lambda_{345} v_{1} v_{2} & m_{12}^{2} \frac{v_{1}}{v_{2}}+\lambda_{2} v_{2}^{2}
\end{array}\right) \\
& \mathcal{M}_{P}=\left(\begin{array}{cc}
\frac{m_{12}^{2}}{v_{1} v_{2}}-\lambda_{5}
\end{array}\right)\left(\begin{array}{cc}
v_{2}^{2} & -v_{1} v_{2} \\
-v_{1} v_{2} & v_{1}^{2}
\end{array}\right) \\
& \mathcal{M}_{C}=\left(\frac{m_{12}^{2}}{v_{1} v_{2}}-\frac{\lambda_{4}+\lambda_{5}}{2}\right)\left(\begin{array}{cc}
v_{2}^{2} & -v_{1} v_{2} \\
-v_{1} v_{2} & v_{1}^{2}
\end{array}\right) .
\end{aligned}
$$

In Eqs. (2.7) and (2.8), we already see that the pseudoscalar and charged scalar matrices have determinant equal to zero- and therefore a zero eigenvalue, corresponding to the expected neutral and charged Goldstone bosons. The diagonalization of the mass matrices is performed via the following orthogonal transformations

$$
\begin{aligned}
& \left(\begin{array}{l}
\rho_{1} \\
\rho_{2}
\end{array}\right)=R(\alpha)\left(\begin{array}{l}
H \\
h
\end{array}\right), \\
& \left(\begin{array}{l}
\eta_{1} \\
\eta_{2}
\end{array}\right)=R(\beta)\left(\begin{array}{l}
G^{0} \\
A
\end{array}\right),
\end{aligned}
$$




$$
\left(\begin{array}{c}
\phi_{1}^{ \pm} \\
\phi_{2}^{ \pm}
\end{array}\right)=R(\beta)\left(\begin{array}{c}
G^{ \pm} \\
H^{ \pm}
\end{array}\right),
$$

where the rotation matrices have the form

$$
R(\vartheta)=\left(\begin{array}{cc}
\cos \vartheta & -\sin \vartheta \\
\sin \vartheta & \cos \vartheta
\end{array}\right)
$$

with $\vartheta=\alpha$ or $\beta$. These rotations lead us to the physical states, which include one neutral $C P$-odd state, $A$, two neutral $C P$-even states, $h$ and $H$, and two charged Higgs bosons, $H^{ \pm}$, besides the longitudinal components of the $W^{ \pm}$and the $Z$ bosons, the pseudo-Nambu-Goldstone bosons $G^{ \pm}$and $G^{0}$, respectively.

The angle $\beta$ can be defined at tree level as

$$
\tan \beta=\frac{v_{2}}{v_{1}},
$$

while $v_{1}^{2}+v_{2}^{2}=v^{2} \approx(246 \mathrm{GeV})^{2}$ ensures the correct pattern of symmetry breaking. The mixing angle $\alpha$ can be written in terms of $\left(\mathcal{M}_{S}\right)_{i j}(i, j=1,2)$, which are the entries of the $C P$-even scalar mass matrix, as

$$
\tan 2 \alpha=\frac{2\left(\mathcal{M}_{S}\right)_{12}}{\left(\mathcal{M}_{S}\right)_{11}-\left(\mathcal{M}_{S}\right)_{22}} .
$$

Introducing the quantity $M$ defined as

$$
M^{2} \equiv \frac{m_{12}^{2}}{s_{\beta} c_{\beta}},
$$

with the short-hand notation $s_{x} \equiv \sin x$ etc., we can write [44]

$$
\tan 2 \alpha=\frac{s_{2 \beta}\left(M^{2}-\lambda_{345} v^{2}\right)}{c_{\beta}^{2}\left(M^{2}-\lambda_{1} v^{2}\right)-s_{\beta}^{2}\left(M^{2}-\lambda_{2} v^{2}\right)} .
$$

Finally, the scalar masses may be written as

$$
\begin{aligned}
m_{h, H}^{2}= & \frac{1}{2}\left[\left(\mathcal{M}_{S}\right)_{11}+\left(\mathcal{M}_{S}\right)_{22}\right. \\
& \left.\mp \sqrt{\left(\left(\mathcal{M}_{S}\right)_{11}-\left(\mathcal{M}_{S}\right)_{22}\right)^{2}+4\left(\left(\mathcal{M}_{S}\right)_{12}\right)^{2}}\right] \\
m_{A}^{2}= & M^{2}-\lambda_{5} v^{2} \\
m_{H^{ \pm}}^{2}= & M^{2}-\frac{\lambda_{4}+\lambda_{5}}{2} v^{2} .
\end{aligned}
$$

The potential has eight independent parameters and we choose: the four scalar masses (the two masses of the $C P$ even states, the mass of the $C P$-odd state and the mass of the charged Higgs boson), the rotation angle in the $C P$ even sector, $\alpha$, the ratio of the vacuum expectation values, $\tan \beta=v_{2} / v_{1}$, the soft breaking parameter $m_{12}^{2}$ and $v^{2}=v_{1}^{2}+v_{2}^{2}$. Without loss of generality, we adopt the conventions $0 \leq \beta \leq \pi / 2$ and $-\pi / 2 \leq \alpha \leq \pi / 2$.

The two doublets $\Phi_{1}$ and $\Phi_{2}$ are not physical fields, unlike the mass eigenstates. This means that any linear combination of the doublets which preserves the form of the kinetic terms of the theory is equally acceptable. This reparametrization freedom implies that different bases of the doublet fields can be chosen, without changing physical predictions of the model and potentially simplifying the theory. It is sometimes useful to work in the so-called Higgs basis, wherein one performs a $U(2)$ transformation on $\Phi_{1}$, $\Phi_{2}$ in such a manner that only the first of the transformed fields, $\left\{H_{1}, H_{2}\right\}$, has a VEV. The Higgs basis may be defined for our model by the rotation ${ }^{2}$

$$
\left(\begin{array}{l}
H_{1} \\
H_{2}
\end{array}\right)=R_{H}\left(\begin{array}{l}
\Phi_{1} \\
\Phi_{2}
\end{array}\right) \equiv\left(\begin{array}{cc}
c_{\beta} & s_{\beta} \\
-s_{\beta} & c_{\beta}
\end{array}\right)\left(\begin{array}{l}
\Phi_{1} \\
\Phi_{2}
\end{array}\right)
$$

and hence the potential can be written as [45]

$$
\begin{aligned}
V\left(H_{1}, H_{2}\right)= & Y_{1} H_{1}^{\dagger} H_{1}+Y_{2} H_{2}^{\dagger} H_{2}-\left(Y_{3} H_{1}^{\dagger} H_{2}+\text { H.c. }\right) \\
& +\frac{1}{2} Z_{1}\left(H_{1}^{\dagger} H_{1}\right)^{2}+\frac{1}{2} Z_{2}\left(H_{2}^{\dagger} H_{2}\right)^{2} \\
& +Z_{3}\left(H_{1}^{\dagger} H_{1}\right)\left(H_{2}^{\dagger} H_{2}\right)+Z_{4}\left(H_{1}^{\dagger} H_{2}\right)\left(H_{2}^{\dagger} H_{1}\right) \\
& +\left\{\frac{1}{2} Z_{5}\left(H_{1}^{\dagger} H_{2}\right)^{2}+\left[Z_{6} H_{1}^{\dagger} H_{1}\right.\right. \\
& \left.\left.+Z_{7} H_{2}^{\dagger} H_{2}\right] H_{1}^{\dagger} H_{2}+\text { H.c. }\right\}
\end{aligned}
$$

with the minimization conditions of the potential in this new basis implying that the parameters $Y_{3}$ and $Z_{6}$ are related to one another.

The reason why we are interested in this form of the potential is that it allows to write expressions that facilitate in some cases the discussion of alignment and decoupling limits in the 2HDM [46]. Let us clarify what we mean by alignment and decoupling: the LHC has shown beyond all doubt that the $125 \mathrm{GeV}$ scalar which has been discovered has SM-like behavior, meaning it seems to couple to gauge bosons and fermions very much like the SM Higgs boson would do. Within models with two doublets, this implies that the scalar state with $125 \mathrm{GeV}$ mass needs to be almost aligned with the VEV. How does one obtain such aligned regimes in the 2HDM? The key issue is looking at the $C P$ even mass matrix from Eq. (2.6): in the Higgs basis, this matrix becomes

\footnotetext{
${ }^{2}$ The Higgs basis is in fact defined up to an arbitrary complex phase multiplying the second doublet.
} 


$$
\mathcal{M}_{S}=\left(\begin{array}{cc}
Z_{1} v^{2} & Z_{6} v^{2} \\
Z_{6} v^{2} & m_{A}^{2}+Z_{5} v^{2}
\end{array}\right) .
$$

Having an aligned scalar means that there won't be much mixing between the two $C P$-even states, and this can be achieved in two ways:

(i) One of the diagonal elements in Eq. (2.20) is much bigger than the other one. Since $Z_{1}$ is a quartic coupling and therefore expected not to be large, this forces the $(2,2)$ entry in the matrix to be quite large, and it is simple to show that all extra scalars will be heavy. In this regime, alignment is achieved in the decoupling limit.

(ii) The off-diagonal elements in Eq. (2.20) are much smaller than the diagonal ones. In this regime, the masses of the extra scalars are not necessarily large, and the SM-like behavior of the $125 \mathrm{GeV}$ state is said to be caused by the alignment limit.

Looking specifically at the couplings of $h$ or $H$ to gauge bosons, the relevant expressions for our discussion are $[33,37]$

$$
\left|s_{\beta-\alpha} c_{\beta-\alpha}\right|=\frac{\left|Z_{6}\right| v^{2}}{m_{H}^{2}-m_{h}^{2}},
$$

and

$$
Z_{1} v^{2}=m_{h}^{2} s_{\beta-\alpha}^{2}+m_{H}^{2} c_{\beta-\alpha}^{2}
$$

with $Z_{1}$ and $Z_{6}$ given in terms of the original parameters of the Lagrangian by [45]

$$
\begin{aligned}
& Z_{1}=\lambda_{1} c_{\beta}^{4}+\lambda_{2} s_{\beta}^{4}+\frac{1}{2} \lambda_{345} s_{2 \beta}^{2}, \\
& Z_{6}=-\frac{1}{2} s_{2 \beta}\left[\lambda_{1} c_{\beta}^{2}-\lambda_{2} s_{\beta}^{2}-\lambda_{345} c_{2 \beta}\right] .
\end{aligned}
$$

Assuming that the lightest state is the one that is aligned with the VEV, and that it has a mass of $125 \mathrm{GeV}$, its treelevel couplings are very close to the SM Higgs ones. This limit is attained by setting $c_{\beta-\alpha} \rightarrow 0$. Equation (2.21) tells us then that it is sufficient to have $Z_{6} \ll 1$ to be in the alignment limit. In this regime, although the couplings of the $125 \mathrm{GeV}$ Higgs are all SM-like, the other Higgs bosons can in principle be light and therefore be within the reach of the LHC. To have alignment in the decoupling limit the masses of the non-125 Higgs bosons must be much larger than $125 \mathrm{GeV}$. Defining a common mass scale $m_{\phi_{\text {heavy }}}$ with $\phi_{\text {heavy }}=H, A$ and $H^{ \pm}$one can write [44]

$$
m_{\phi_{\text {heavy }}}^{2}=M^{2}+f\left(\lambda_{i}\right) v^{2}+\mathcal{O}\left(v^{4} / M^{2}\right),
$$

where $f\left(\lambda_{i}\right)$ denotes a linear combination of $\lambda_{1} \ldots \lambda_{5}{ }^{3}$ If $M^{2} \gg f\left(\lambda_{i}\right) v^{2}$ all masses are of the order of $M$ and therefore quite large - and from Eq. (2.21) again we obtain $|\cos (\beta-\alpha)| \simeq 0$.

In the case $s_{\beta-\alpha} \rightarrow 0$ there is again alignment but now with the heavy $C P$-even Higgs $H$, meaning this would correspond to the heavy Higgs scenario mentioned above. The condition for this regime to occur is still $Z_{6} \ll 1$, but now decoupling is not possible, as the non-SM-like Higgs boson masses are not all much larger than $125 \mathrm{GeV}$, in particular not $m_{h}$.

\section{THEORETICAL CONSTRAINTS ON THE PARAMETRERS}

The main goal of this study is to understand the effects of the RGE evolution of the couplings, from the weak scale (the mass of the $Z$ boson, $m_{Z}$ ) to higher scales all the way up to the Planck scale, $\Lambda=10^{19} \mathrm{GeV}$. Our procedure consists in first inputting a set of $2 \mathrm{HDM}$ parameters at the weak scale and verifying whether they satisfy the following theoretical demands:

(i) The potential is bounded from below, so that the theory is guaranteed to have a stable vacuum of some sort.

This is achieved by demanding that the quartic couplings of the potential obey $[47,48]$

$$
\begin{aligned}
& \lambda_{1}>0, \quad \lambda_{2}>0, \\
& \lambda_{3}>-\sqrt{\lambda_{1} \lambda_{2}}, \quad \lambda_{3}+\lambda_{4}-\left|\lambda_{5}\right|>-\sqrt{\lambda_{1} \lambda_{2}} .
\end{aligned}
$$

These conditions have been shown to be necessary and sufficient [49] to ensure that the scalar potential is bounded from below (in the "strong sense" as defined in Refs. [50,51]).

(ii) That the minimum is global and provides the right pattern of electroweak symmetry breaking.

Contrary to the SM, the 2HDM can have several simultaneous stationary points. Besides the $C P$ conserving minimum, the model can have $C P$ violating $(\mathrm{CPV})$ and Charge Breaking $(\mathrm{CB})$ minima, which are spontaneously generated. As shown in [40,49,52,53], if the potential is in a $C P$-conserving minimum, any other stationary point, if of a different nature (either $\mathrm{CPV}$ or $\mathrm{CB}$ ), is a saddle point with higher value of the potential. Still, there is a possibility that two $C P$-conserving minima could co-exist. In this case tunnelling could occur from our minimum to another one with a different electroweak scale. In $[54,55]$ this minimum was called the panic vacuum. However, it was found that verifying if the parameters of the potential obey a simple

\footnotetext{
${ }^{3}$ In fact, we see from Eq. (2.17) that for $m_{A}$ and $m_{H^{ \pm}}$the $v^{4}$ terms are not even present.
} 
condition [54-56] it is possible to know exactly whether our $C P$-conserving vacuum is the global one. We define the discriminant

$$
D=m_{12}^{2}\left(m_{11}^{2}-k^{2} m_{22}^{2}\right)\left(\frac{v_{2}}{v_{1}}-k\right)
$$

where $k=\sqrt[4]{\lambda_{1} / \lambda_{2}}$, and the VEVs are the ones that define the correct pattern of symmetry breaking (meaning, they predict the correct gauge boson and fermion masses, $\left.v_{1}^{2}+v_{2}^{2}=(246 \mathrm{GeV})^{2}\right)$. The existence of a panic vacuum is thus summarized in the following theorem:

The vacuum with the correct pattern of symmetry breaking is the global minimum of the potential if and only if $D>0$.

(iii) That perturbative unitarity ${ }^{4}$ holds.

We enforce tree-level perturbative unitarity by requiring that the eigenvalues of the $2 \rightarrow 2$ scalar scattering matrix are below $8 \pi$ [57]. The full $2 \rightarrow 2$ scattering matrix of the fields in the gauge basis has been computed [57] (see also [58,59]), and its eigenvalues are

$$
\begin{aligned}
& b_{ \pm}=\frac{1}{2}\left(\lambda_{1}+\lambda_{2} \pm \sqrt{\left(\lambda_{1}-\lambda_{2}\right)^{2}+4 \lambda_{5}^{2}}\right) \\
& c_{ \pm}=\frac{1}{2}\left(\lambda_{1}+\lambda_{2} \pm \sqrt{\left(\lambda_{1}-\lambda_{2}\right)^{2}+4 \lambda_{4}^{2}}\right) \\
& e_{1}=\lambda_{3}+2 \lambda_{4}-3 \lambda_{5} \\
& e_{2}=\lambda_{3}-\lambda_{5} \\
& f_{+}=\lambda_{3}+2 \lambda_{4}+3 \lambda_{5} \\
& f_{-}=\lambda_{3}+\lambda_{5} \\
& f_{1}=\lambda_{3}+\lambda_{4} \\
& p_{1}=\lambda_{3}-\lambda_{4} .
\end{aligned}
$$

In this calculation, only the eigenvalues of the two-particle scattering matrix were consideredall two-particle states made of scalars, either physical (the five Higgs bosons) or unphysical (the Goldstone bosons) were included in the calculation. The above eigenvalues are not all independent. As noted in [57],

$$
\begin{gathered}
3 f_{1}=p_{1}+e_{1}+f_{+} \\
3 e_{2}=2 p_{1}+e_{1} \\
3 f_{-}=2 p_{1}+f_{+} .
\end{gathered}
$$

\footnotetext{
${ }^{4}$ We note that a model that does not respect perturbative unitarity is not necessarily wrong. However, discussing this possibility is beyond the scope of this work.
}

This means that the conditions on $f_{1}, e_{2}$ and $f_{-}$ can be dropped. Moreover, adding the fact that $\lambda_{1}$, $\lambda_{2}>0$ is needed for the potential to be bounded from below, we obtain

$$
\begin{aligned}
& \left|c_{+}\right|>\left|c_{-}\right| \\
& \left|b_{+}\right|>\left|b_{-}\right| .
\end{aligned}
$$

The resulting conditions for tree-level perturbative unitarity are thus given by

$$
\begin{aligned}
\left|\lambda_{3}-\lambda_{4}\right| & <8 \pi \\
\left|\lambda_{3}+2 \lambda_{4} \pm 3 \lambda_{5}\right| & <8 \pi \\
\left|\frac{1}{2}\left(\lambda_{1}+\lambda_{2}+\sqrt{\left(\lambda_{1}-\lambda_{2}\right)^{2}+4 \lambda_{4}^{2}}\right)\right| & <8 \pi \\
\left|\frac{1}{2}\left(\lambda_{1}+\lambda_{2}+\sqrt{\left(\lambda_{1}-\lambda_{2}\right)^{2}+4 \lambda_{5}^{2}}\right)\right| & <8 \pi .
\end{aligned}
$$

If a given choice of $2 \mathrm{HDM}$ parameters satisfies all of these constraints, it is accepted (provided it further satisfies, at the weak scale, the experimental constraints described in the next section). At this stage we include the effect of the renormalization group running of the parameters of the theory to understand how it affects the allowed parameter space. We use the one-loop $\beta$ functions for the parameters of the model (and also the VEVs $v_{1}$ and $v_{2}$ ), presented in Appendix A, and for each point in the parameter space chosen. We adopt the following procedure:

(i) Perform the RGE running of all potential parameters and VEVs starting at $m_{Z}$.

(ii) At each scale between $m_{Z}$ and the Planck scale, verify whether the theoretical constraints detailed above (potential bounded from below; positive discriminant; perturbative unitarity) are still verified. $^{5}$

(iii) If all the theoretical constraints are verified, proceed to a higher scale and repeat.

(iv) If at a given scale $\Lambda$ any of the theoretical constraints is not verified, stop the RGE running and keep the information on this cutoff scale.

There is a further constraint which must be considered - for a large region of the initial parameter space, the RGE running will hit Landau poles-i.e., the parameters will tend to infinity - at some scale between $m_{Z}$ and the Planck scale. As in the SM, this is easily understood if one considers the structure of the couplings in the $\beta$ functions of the model. For instance, the contributions of the Yukawa couplings to the $\beta$ functions of the quartic couplings are negative and tend to reduce their values as one increases the renormalization scale; but the quartic couplings have

\footnotetext{
${ }^{5}$ An alternative approach, which enlarges the parameter space considered, was advocated in Ref. [60].
} 
positive contributions to those $\beta$ functions and thus tend to increase their values. As a consequence, only initial values of the quartic couplings with small magnitudes will not develop Landau poles during the RGE running up to the Planck scale. For completion, we assume a Landau pole occurs if either (a) one of the gauge couplings, Yukawa couplings or quartic couplings of the potential reaches the absolute value of 100 or (b) if either $m_{11}^{2}, m_{22}^{2}$ or $m_{12}^{2}$ reaches the absolute value of $10^{10} \times v^{2}(v=246 \mathrm{GeV})$ or (c) if $v_{2} / v_{1}>100$. Notice that since the $\beta$ functions are highly coupled, as soon as one given parameter hits a Landau pole typically others will as well.

If some choice of parameters is such that one theoretical constraint is violated or a Landau pole occurs at a given scale $\Lambda$, this means that the theory ceases to be valid above $\Lambda$ and requires new physics (NP) above $\Lambda$ to correct the RGE evolution (for example, extra scalars to stabilize the vacuum, or extra fermions to prevent Landau poles). Thus, if one believes that the 2HDM should be valid up to a given high-energy scale $\Lambda_{N P}$, the RGE running described will discard many combinations of parameters, reducing the parameter space of the model and improving its predictability. The higher $\Lambda_{N P}$ is the more severe is the elimination of parameters. To give the reader an idea of the importance of each of our requirements on the curtailment of the 2HDM parameter space, we found that the appearance of a Landau pole at $\Lambda_{N P}=1 \mathrm{TeV}$ reduces the number of original points to about $46 \%$ in model type I, and $33 \%$ in model type II $^{6}$ requiring the potential to also be bounded from below and unitarity to be obeyed up to the same scale of $1 \mathrm{TeV}$ will amount to a further reduction to $17 \%$ (8\%) for type I (type II) of the original points. Finally, the discriminant plays a very small role, with a further reduction of less than $1 \%$ for again a scale of $1 \mathrm{TeV}$, for both model types. In fact, the discriminant will almost play no role in this analysis-the number of points which do not survive RGE running all the way to the Planck scale because only the discriminant condition is violated is extremely small.

Finally, a word on thresholds: we have taken the weak scale, $m_{Z}$, as the starting point of our RG analysis. A more refined analysis would take into account the possibility of thresholds in the RG running (for instance, using the 5-flavor $\beta$ functions between $m_{Z}$ and the top threshold). Alternatively, we could have started the RG running at a higher scale. In either case, the impact of these refinements in the RG running in our analysis is minimal, at most slightly shifting the cutoff scales $\Lambda$. The substance of our conclusions would not be affected.

\footnotetext{
${ }^{6}$ The $\beta$ functions are, of course, different for each of the model types considered; see Appendix A.
}

\section{THE 2HDM PARAMETER SPACE}

The 2HDM is implemented as a model class in Scanners [61,62], and we used this code to generate our data samples. The theoretical bounds described in the previous section, plus all relevant experimental constraints (available at the time of write-up), are either inbuilt in the code or interfaces with several other codes allow to take them into account in the sample generation.

We will now briefly describe the experimental constraints on the model and how they are applied. The most relevant exclusion bounds on the $m_{H^{ \pm}}-t_{\beta}$ plane are those which arise from the $B \rightarrow X_{s} \gamma$ measurements [63-67]. A $2 \sigma$ bound on the charged Higgs mass of $m_{H^{ \pm}}>580 \mathrm{GeV}$ for the models type II and Flipped that is almost independent of $\tan \beta$ was recently discussed in [67]. In all types of 2HDMs, there is also a hard bound on the charged Higgs mass coming from LEP, with the process $e^{+} e^{-} \rightarrow H^{+} H^{-}$ [68] which is approximately $100 \mathrm{GeV}$. We have used all the flavor constraints, plus the ones from the $R_{b}[63,69]$ measurement. These constraints are $2 \sigma$ exclusion bounds on the $m_{H^{ \pm}}-t_{\beta}$ plane. Furthermore, all points comply with the electroweak precision measurements. We demand a $2 \sigma$ compatibility of the $S, T$ and $U$ parameters with the SM fit presented in [70]. The full correlation among these parameters is taken into account.

The mass of the SM-like Higgs boson, denoted by $h_{125}$, is set to $m_{h_{125}}=125.09 \mathrm{GeV}$ [71]. The HiggsBounds 4.3.1 (the latest stable version) code [72-74] is used to check for agreement with all $2 \sigma$ exclusion limits from LEP, Tevatron and LHC Higgs searches. The decay widths and branching ratios were calculated with HDECAY [75,76], which includes off-shell decays and state-of-the-art QCD corrections. All Higgs boson production cross sections via gluon fusion $(g g F)$ and $b$-quark fusion $(b b F)$ are obtained from SusHiv1.6.0 [77,78], at NNLO QCD. The SMlike Higgs rates are forced to be within $2 \times 1 \sigma$ of the fitted experimental values given in [79]. In that reference, bounds are presented for the quantities

$$
\frac{\mu_{F}}{\mu_{V}}, \quad \mu_{\gamma \gamma}, \quad \mu_{Z Z}, \quad \mu_{W W}, \quad \mu_{\tau \tau}, \quad \mu_{b b},
$$

where $\mu_{F}\left(\mu_{V}\right)$ is the ratio, for each channel, between the measured cross section, and its SM expected value, for the gluon-gluon fusion and $t \bar{t} H(\mathrm{VBF}+\mathrm{VH})$ production processes; the quantities $\mu_{x x}$ are then defined as

$$
\mu_{x x}=\mu_{F} \frac{\mathrm{BR}_{2 \mathrm{HDM}}\left(H_{i} \rightarrow x x\right)}{\mathrm{BR}_{\mathrm{SM}}\left(H_{\mathrm{SM}} \rightarrow x x\right)}
$$

for $H_{i} \equiv h_{125}$ and the SM Higgs boson $H_{\mathrm{SM}}$. Because custodial symmetry is preserved, $\mu_{Z Z}=\mu_{W W} \equiv \mu_{V V}$, and we are allowed to combine the lower $2 \times 1 \sigma$ bound from $\mu_{Z Z}$ with the upper bound on $\mu_{W W}$ [79], 


$$
0.79<\mu_{V V}<1.48
$$

In type II, we choose the charged Higgs mass to be in the range

$$
580 \mathrm{GeV} \leq m_{H^{ \pm}}<1 \mathrm{TeV},
$$

while in type I, we have taken

$$
80 \mathrm{GeV} \leq m_{H^{ \pm}}<1 \mathrm{TeV} .
$$

We have performed a uniformly distributed random sampling.

Taking into account all the constraints, in order to optimize the scan, we have performed a sampling in the following regions in the remaining input parameters: $0.8 \leq \tan \beta \leq 35,-\frac{\pi}{2} \leq \alpha<\frac{\pi}{2}, 0 \mathrm{GeV}^{2} \leq m_{12}^{2}<500000 \mathrm{GeV}^{2}$, $30 \mathrm{GeV} \leq m_{A}<1000 \mathrm{GeV}$ and finally $130 \leq m_{H}<$ $1000 \mathrm{GeV}$ for the light Higgs scenario, but $30<m_{h}<$ $120 \mathrm{GeV}$ for the heavy Higgs scenario. The sampling of values was performed in a uniformly distributed random way before applying all the constraints.

\section{THE LIGHT HIGGS SCENARIO}

In this section, we will consider the standard approach to the 2HDM, in which the lightest $C P$-even scalar is taken to be the observed $125 \mathrm{GeV}$ Higgs boson. The heavy Higgs scenario is dealt with in the next session. Our goal now is to carefully analyse what impact the requirement of imposing the theoretical constraints described in the previous section has, plus the absence of Landau poles, for all scales above the weak scale.

\section{A. Results with no collider bounds}

We start the discussion with a sample of points that have passed all the theoretical constraints, the electroweak precision tests and all $B$-physics constraints-the most

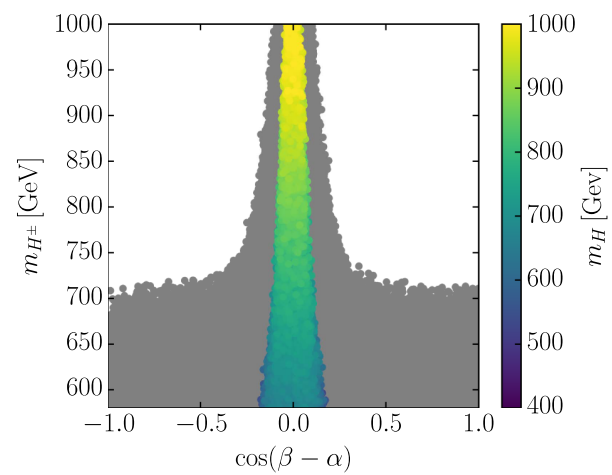

important one being the constraint from $b \rightarrow s \gamma$, which in type II forces the charged Higgs mass to be above $580 \mathrm{GeV}$ at $2 \sigma$. However, we have not imposed the LHC bounds on the observed Higgs rates from [79] on this parameter sample. What we will observe is that the requirement that the potential is well-behaved for increasingly high-energy scales will curtail the parameter space so much that, in some situations, the $125 \mathrm{GeV}$ scalar becomes "naturally" aligned.

Let us begin with the analysis of the type II model. The data sample we used had almost 1 million different parameter combinations, and for each of those points we performed the RGE running described above, verifying the cutoff scales $\Lambda$ for which either the theoretical constraints we imposed were violated or a Landau pole was reached. The results of this work allowed us to obtain Fig. 1, which we now analyze in detail.

On the left panel of Fig. 1 we present the charged Higgs mass vs $\cos (\beta-\alpha)$. We show in grey the points that passed the theoretical, $S, T, U$ and $B$-physics constraints. Notice how clearly the LHC bounds were not present in the initial sample, since $\cos (\beta-\alpha)$ varies from -1 to 1 , whereas current experimental results point to the observed Higgs having SM-like behavior, which would necessitate values of $|\cos (\beta-\alpha)|$ much closer to zero. The colored points in the plot are the subset of the initial data sample which survived the RGE running up to a scale of $1 \mathrm{TeV}-$ meaning, for which no theoretical constraint was violated, nor a Landau pole occurred, between $m_{Z}$ and $1 \mathrm{TeV}$. The color bar shows the minimum value of $m_{H}$, which shows a similar trend to the charged Higgs mass. For example, with the cutoff scale of $1 \mathrm{TeV}$, the minimum value of $m_{H}$ allowed is about $440 \mathrm{GeV}$. It is clear that, already at such a small scale as $1 \mathrm{TeV}$, the range of variation of $\cos (\beta-\alpha)$ has shrunk from the original $|\cos (\beta-\alpha)|<1$ to about $|\cos (\beta-\alpha)|<0.2$ - which means that the simple requirement that the type II potential is well behaved up to a scale of about $1 \mathrm{TeV}$ implies that the Higgs boson must have

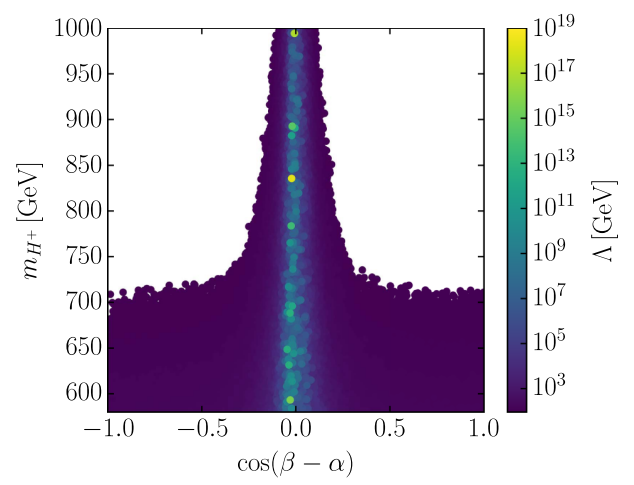

FIG. 1. Charged Higgs mass vs $\cos (\beta-\alpha)$ in the type II 2HDM. On the left panel we show, in grey, the points that passed the theoretical, electroweak and $B$-physics constraints at the scale $m_{Z}$. The remaining points have survived the RGE running up to a scale of $1 \mathrm{TeV}$. The color bar shows the value of $m_{H}$. On the right panel we present the same plot but where the color bar shows the cutoff scale. The points are sorted from dark to brighter colors. 
SM-like behaviour provided that one of the scalars has a mass above $\approx 500 \mathrm{GeV}$. Here we see alignment arising in a "natural" way from the behavior of the theory, rather than requiring a particular choice of the parameter region to fit the data.

On the right panel of Fig. 1 we show the result of continuing the RGE running for higher scales than $1 \mathrm{TeV}$. Again we plot $m_{H^{ \pm}}$vs $\cos (\beta-\alpha)$, but now the color code provides information on the cutoff scale $\Lambda$, that is, the scale at which either a Landau pole occurs or any of the theoretical conditions is violated. As expected from previous analyses, many points survive up to the Planck scale with values of $\cos (\beta-\alpha)$ increasingly closer to zero. The $2 \mathrm{HDM}$ of type II can therefore be a valid description of particle physics all the way up to the Planck scale-and since we made sure the potential is bounded from below and the correct electroweak minimum is the global one at all scales, we conclude that within the framework of this model it is possible to choose parameters to even avoid the issue of metastability which has been discussed for the SM.

What is the origin of the quick approach of the alignment regime, at such a remarkably low energy scale as $1 \mathrm{TeV}$ ? In Fig. 2 we present the points that have survived the running up to the cutoff scale $\Lambda$. On the left we see that $M$ has a very fast increase, reaching a maximum value of $423 \mathrm{GeV}$ at $1 \mathrm{TeV}$. For higher scales we see that $M$ can take many values, but its minimum value then stabilizes, with a value close to the charged Higgs mass at $\Lambda \approx 10^{3} \mathrm{TeV}$, and remains constant up to the Planck scale.

On the right panel of Fig. 2 we show the values of $\lambda_{4}+\lambda_{5}$ for the points which survive from $m_{Z}$ up to the Planck scale. Clearly, the absolute value of $\lambda_{4}+\lambda_{5}$ is decreasing, and the sum of the two couplings can only take values close to zero if the model is to be valid up to very high-energy scales. Indeed, already at $1 \mathrm{TeV}$ we have $\left|\lambda_{4}+\lambda_{5}\right| \lesssim 5.7$ and we can attempt an analytical explanation for the approach to alignment, using

$$
M^{2}-m_{H^{ \pm}}^{2}=-\frac{\lambda_{4}+\lambda_{5}}{2} v^{2} \Rightarrow M-m_{H^{ \pm}}=-\frac{\left(\lambda_{4}+\lambda_{5}\right) v^{2}}{2\left(M+m_{H^{ \pm}}\right)} .
$$

Inserting the maximum value for $\lambda_{4}+\lambda_{5}$ and the minimum value for $m_{H^{ \pm}}$and $M$ for $1 \mathrm{TeV}$ in these formulae, we obtain $\left|M-m_{H^{ \pm}}\right| \approx 163 \mathrm{GeV}$. This provides an approximation for the maximum mass difference between the several scalars. To reinforce this point, in Fig. 3 we plot $m_{A}-m_{H^{ \pm}}$ vs $m_{H}-m_{H^{ \pm}}$in the type II 2HDM. Again, the grey points have passed all theoretical constraints and comply with $b \rightarrow s \gamma$ at the scale $m_{Z}$. On the left plot, the remaining points, color coded with the values of $\tan \beta$, have survived the RGE running up to a scale of $1 \mathrm{TeV}$. As discussed, we can see clearly in the plot that all mass differences are below $\pm 200 \mathrm{GeV}$ - and since in type II the charged Higgs mass is constrained by the $b \rightarrow s \gamma$ results to be above $580 \mathrm{GeV}$, this gives us possible lower bounds on the masses for the pseudoscalar or the heavier $C P$-even scalar of about $430 \mathrm{GeV}$.

Let us now consider the right plot of Fig. 3, where we can see the dependence of the cutoff scale. Because the absolute values of the quartic couplings decrease and the lowest value of $M$ stabilizes with increasing cutoff scale, the mass differences approach zero. As the quartic couplings decrease, then, all masses are increasingly controlled by $M$ and they tend to be of the same order. Thus, validity at very high scales $(\gg 1 \mathrm{TeV}$ ) implies, for model type II, that all extra scalars have necessarily high masses, and alignment is reached via decoupling - we found the minimum acceptable values for the scalar masses if the model is valid above $10^{11} \mathrm{GeV}$ is roughly $600 \mathrm{GeV}$. However, if one is more conservative and only assumes that the 2HDM describes particle physics up to a scale of about $1 \mathrm{TeV}$, then alignment can be reached with relatively low masses (450 GeV) for $A$ and $H$, and one could argue that we are
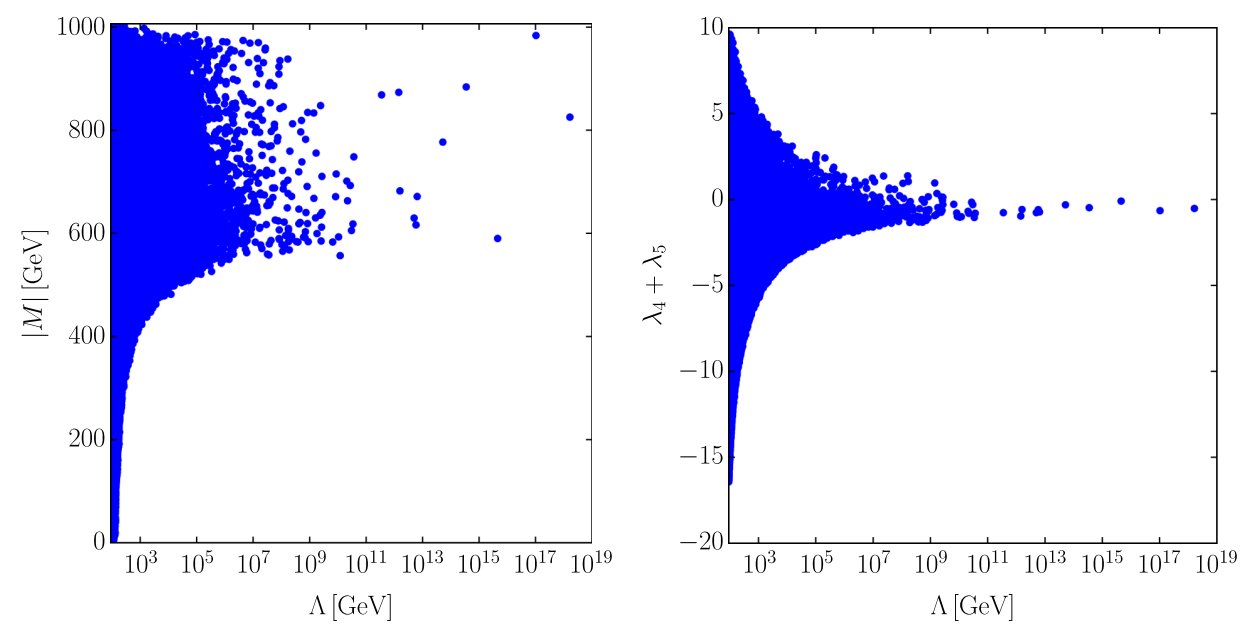

FIG. 2. On the left (right) panel we present $|M|\left(\lambda_{4}+\lambda_{5}\right)$ as a function of the cutoff scale $\Lambda$ in the type II 2 HDM. The points have passed both the theoretical constraints and $b \rightarrow s \gamma$ at the scale $m_{Z}$ and have also survived the RGE running up to the cutoff scale. 

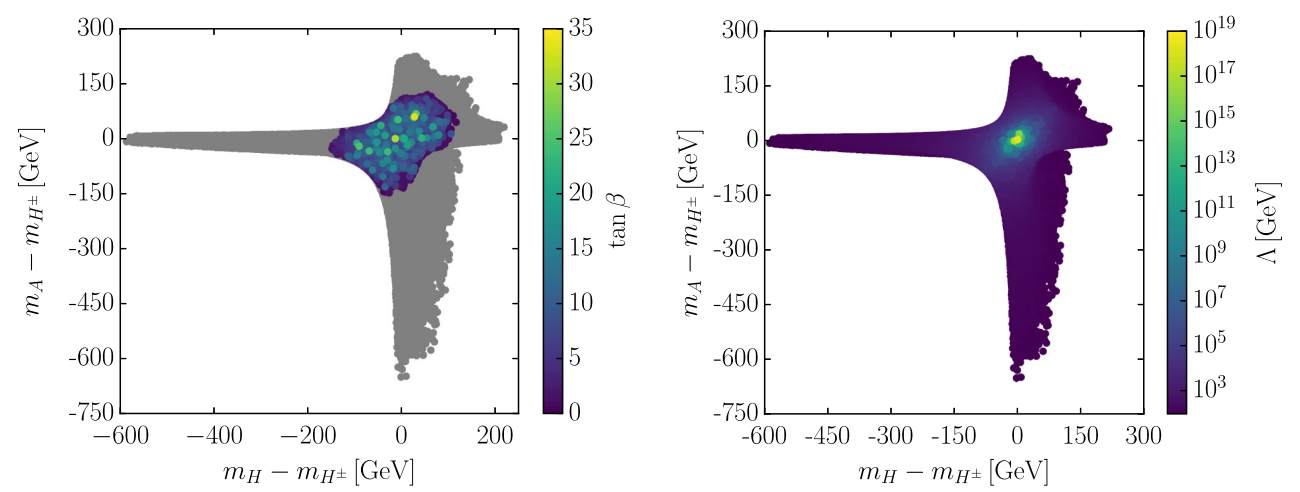

FIG. 3. $m_{A}-m_{H^{ \pm}}$vs $m_{H}-m_{H^{ \pm}}$in the type II 2HDM. On the left panel we show in grey the points that passed the theoretical, electroweak and $B$-physics constraints at the scale $m_{Z}$. The remaining colored points survived the RGE running up to a scale of 1 TeV. The color bar shows the values of $\tan \beta$. On the right panel we present the same plot but where the color bar shows the cutoff scale.

observing the alignment limit, instead of the decoupling limit. In either case, though, the result is the same: requiring the type II $2 \mathrm{HDM}$ to be well behaved at least up to a scale of $1 \mathrm{TeV}$ automatically means that alignment must be satisfied.

The situation is, however, different for a $2 \mathrm{HDM}$ of type I. In Fig. 4 we show the analog, for type I, of Fig. 1. And the striking difference is that requiring the validity of the model up to high energy scales does not necessarily imply alignment for the lightest Higgs boson-we see, in the plot of the right, plenty of points away from alignment (with large absolute values of $\cos (\beta-\alpha)$ ) which survive all the way up to the Planck scale. Thus validity of the 2HDM up to high-energy scales does not necessarily imply alignment for type I, though it does for type II. The left plot of Fig. 4 shows that the charged Higgs mass is playing a crucial role in this respect-in fact, if in model type I one were to impose $m_{H^{ \pm}}>500 \mathrm{GeV}$, again one would have alignment emerging from requiring that the model be valid up to energy scales of at least $1 \mathrm{TeV}$. However, for model type I there is no compelling physics reason to impose such a cut on the charged mass, unlike what happens in type II. Still, the reasoning can be inverted: if particle physics is described by a type I $2 \mathrm{HDM}$, the fact that LHC indicates that the lightest scalar is aligned means that, for the model to be valid up to very high-energy scales, the "natural" expectation is to have a charged Higgs mass superior to $500 \mathrm{GeV}$. This may be understood from the right plot in Fig. 4-points where validity occurs up to the Planck scale with lower charged masses are certainly possible, but not necessarily aligned. Thus, if the charged mass is below roughly $500 \mathrm{GeV}$, validity of the model up to the Planck scale is possible, but alignment does not arise "naturally"; it needs to be further imposed on the model, as a fine-tuning of its parameters. If $m_{H^{ \pm}}>500 \mathrm{GeV}$, on the other hand, validity up to scales as low as $\sim 1 \mathrm{TeV}$ already implies alignment.

\section{B. Results with collider bounds}

In the previous section, we showed how alignment arises, in type II, from requiring that the $2 \mathrm{HDM}$ be valid up to high-energy scales. In type I, alignment does not arise automatically from that requirement, unless one further demands that the charged Higgs mass be superior to $500 \mathrm{GeV}$. Let us now see what the requirement of validity
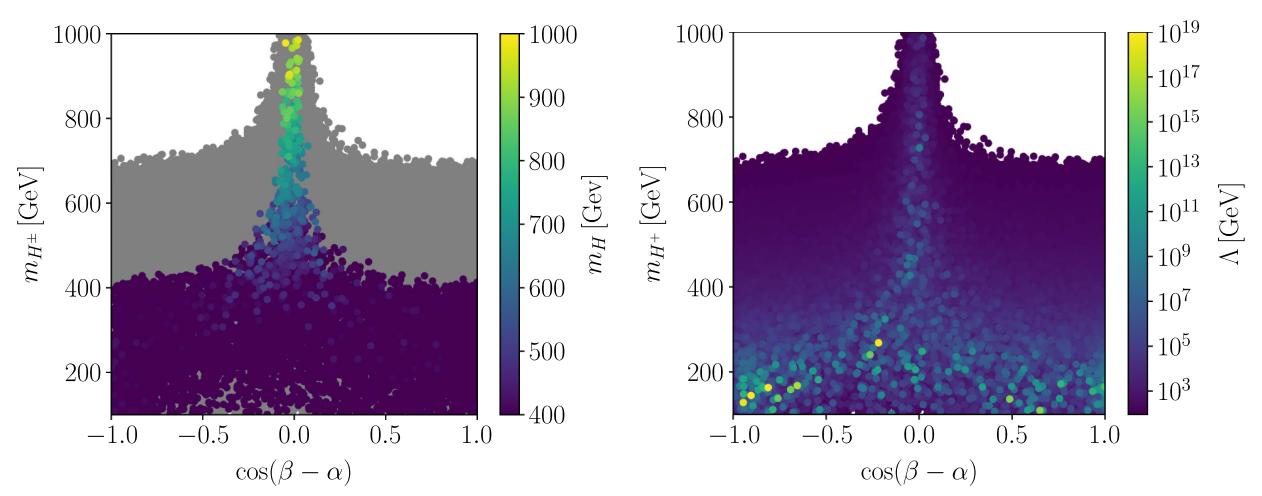

FIG. 4. Charged Higgs mass vs $\cos (\beta-\alpha)$ in the type I 2HDM. On the left panel we show, in grey, the points that passed the theoretical, $S, T, U$ and $B$-physics constraints at the scale $m_{Z}$. The remaining points have survived the RGE running up to a scale of $1 \mathrm{TeV}$. The color bar shows the value of $m_{H}$. On the right panel we present the same plot but where the color bar shows the cutoff scale. 

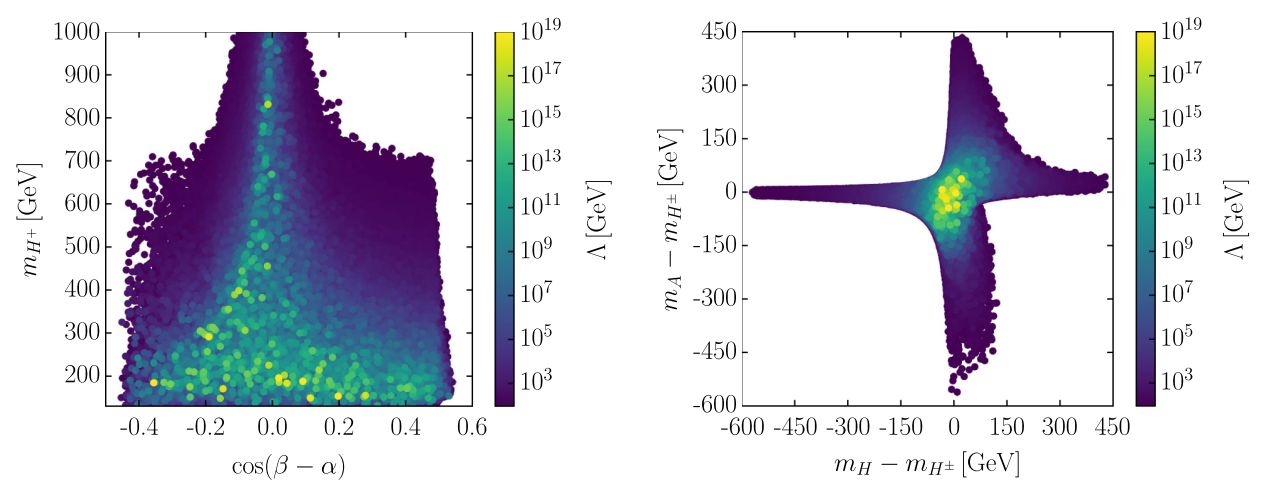

FIG. 5. On the left panel we present the charged Higgs mass vs $\cos (\beta-\alpha)$ in the type I 2HDM, color coded with the cutoff scale. The points have passed all constraints at the scale $m_{Z}$ and have survived up to a given cutoff scale. On the right we present the plot for the mass differences $m_{A}-m_{H^{ \pm}}$vs $m_{H}-m_{H^{ \pm}}$as a function of the cutoff scale.

to high-energy scales combined with the LHC bounds on Higgs physics teaches us about 2HDM phenomenology. In this section, we have used a sample where, besides all theoretical constraints, electroweak precision bounds and constraints from $B$-physics, we have imposed all available collider bounds, and in particular those that restrict the Higgs couplings to fermions and gauge bosons, which are the most relevant ones. The further constraints arising from requiring validity of the model to high scales will obviously increase the predictability of the theory.

A first observation to take into account is that LHC collider bounds are a lot less restrictive for the 2HDM type I than for type II (see, for instance, [80,81]). Even with the latest run II data, the allowed parameter space permits substantial deviations from alignment. We start by presenting in Fig. 5 (left) a plot for the type I $2 \mathrm{HDM}$ in the charged Higgs mass vs $\cos (\beta-\alpha)$ plane. As previously discussed, because there are no strong bounds on the charged Higgs mass, nor on any other scalar besides the $125 \mathrm{GeV}$ one, there is no major difference in the allowed range of $\cos (\beta-\alpha)$ for low and high scales of validity of the theory. This means that, whatever the collider bounds on the type I models are, the model may be valid up to the Planck scale even with large deviations from the alignment limit. Notice the yellow points in the left plot of Fig. 5 with charged Higgs masses as low as $\sim 150 \mathrm{GeV}$ and large absolute values of $\cos (\beta-\alpha)$, corresponding to $2 \mathrm{HDM}$ type I parameter sets for which the model is valid up to the Planck scale — and while not satisfying alignment, they still satisfy all existing LHC bounds.

Still, we would recover the type II results if we had the same bound on the charged Higgs mass as for type IIonce again, if the charged mass is superior to $500 \mathrm{GeV}$, alignment is an automatic consequence of requiring validity of the model up to high scales. On the right plot, we present $m_{A}-m_{H^{ \pm}}$vs $m_{H}-m_{H^{ \pm}}$as a function of the cutoff scale. As for the type II model, the mass differences become increasingly smaller with increasing $\Lambda$, which again suggests that they are controlled by the scale $M$ especially when we move closer to the Planck scale where the quartic couplings are extremely small in magnitude. The reason why we are not driven to decoupling in the generic type I is shown in Fig. 6. Contrary to type II, and although the value of $M$ increases also very fast, its lowest value stabilizes at a value close to $100 \mathrm{GeV}$. Since for high values of the cutoff scale $\Lambda$ the quartic couplings are very close to zero, their contribution to the masses is negligible when compared to that of $M$ (see Eq. (2.17). Thus, light masses, of order $100-200 \mathrm{GeV}$, are still allowed even if the type I model is valid up to high scales.

This leads us to a discussion on the alignment limit, given the plots shown in Fig. 7. There we plot the values of the $Z_{6}$ Higgs-basis coupling (defined in Eqs. (2.19) and (2.23), for both type I and type II models, vs $\cos (\beta-\alpha)$. The "cloud" of points in the type II plot, with large values of $\cos (\beta-\alpha)$, corresponds to the wrong sign limit in that model, to be discussed shortly. As can be seen in the left plot of Fig. 7, values of $Z_{6}$ very close to zero are possible,

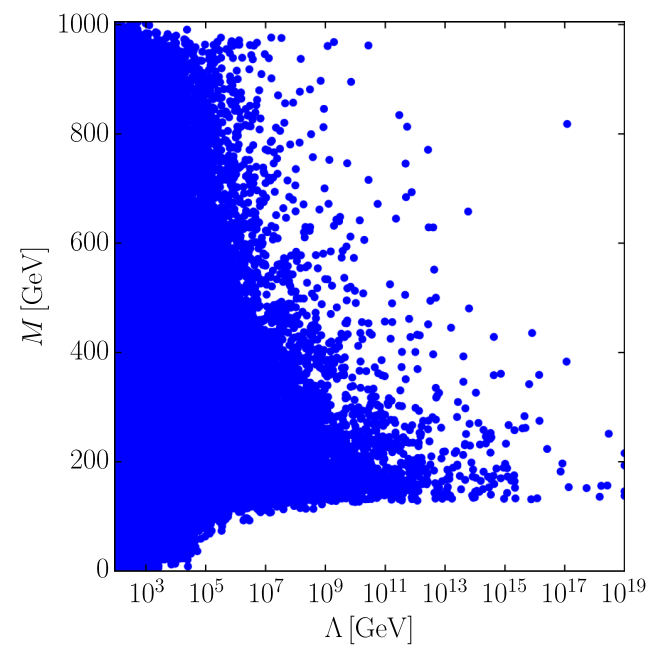

FIG. 6. $\quad M$ as a function of the cutoff scale $\Lambda$ for type I. These points fulfil all experimental and theoretical bounds. 

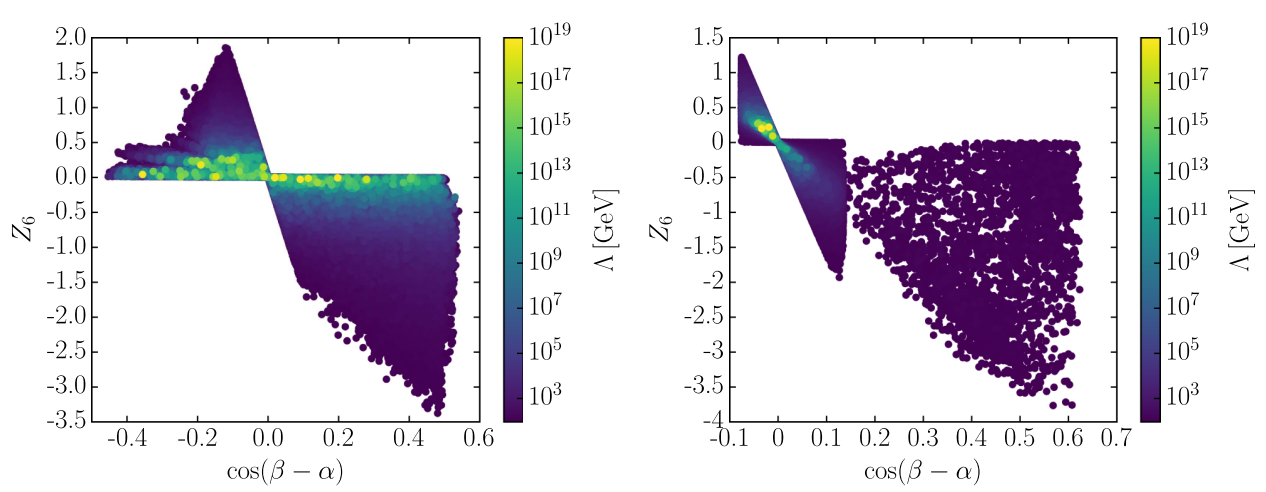

FIG. 7. $Z_{6}$ as a function of $\cos (\beta-\alpha)$ in type I (left) and type II (right) color coded with the cutoff scale.

particularly with the model valid up to the Planck scale. However, even with $Z_{6}$ of very low magnitude, $\cos (\beta-\alpha)$ clearly still varies in a very wide range, and therefore we are not in the alignment limit. Notice the striking difference with the same plot for model type II-there, alignment indeed corresponds to small $Z_{6}$. The difference in behavior between the two models is clearly due to the lower bound on the charged Higgs mass. Therefore, very small values of the quartic couplings may not be enough to make us reach the alignment limit - although for the type II model small $Z_{6}$ is indeed sufficient for alignment, the same cannot be said for type I. For this model, small $Z_{6}$ needs to be complemented by a large enough bound on the charged Higgs mass so that one reaches alignment. We therefore would argue that the alignment limit condition $\left|Z_{6}\right| \ll 1$ is a necessary condition, albeit not a sufficient one. Still, there are certainly values of $Z_{6}$ closer to zero for which alignment would occur independently of the values of the scalar masses, because the matrix in Eq. (2.20) becomes very nearly diagonal for increasingly smaller $Z_{6}$.

In previous works $[81,82]$ two of the authors have discussed the wrong-sign limit of the 2HDM. We define

$$
\kappa_{i}^{2}=\frac{\Gamma^{2 \mathrm{HDM}}(h \rightarrow i)}{\Gamma^{\mathrm{SM}}(h \rightarrow i)}
$$

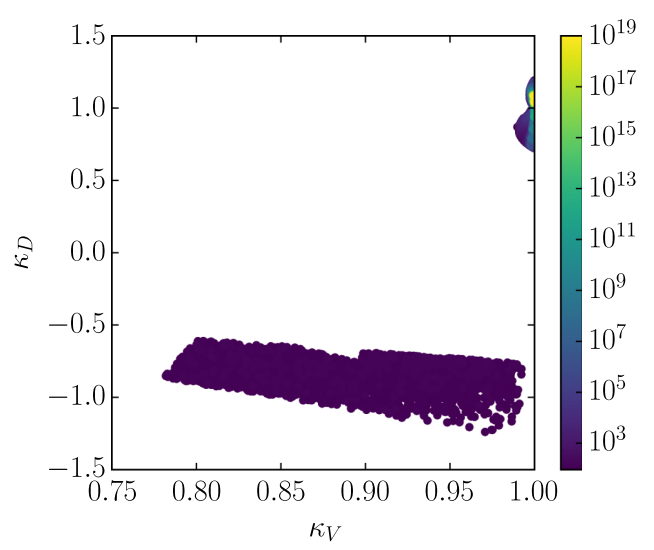

which at tree level is just the ratio of the couplings $\kappa_{i}=g_{i}^{2 \mathrm{HDM}} / g_{i}^{\mathrm{SM}}$ and for the $h W^{+} W^{-}$coupling reads

$$
\kappa_{W}^{2}=\frac{\Gamma^{2 \mathrm{HDM}}\left(h \rightarrow W^{+} W^{-}\right)}{\Gamma^{\mathrm{SM}}\left(h \rightarrow W^{+} W^{-}\right)}=\left(\frac{g_{h W^{+} W^{-}}^{2 \mathrm{HDM}}}{g_{h W^{+} W^{-}}^{\mathrm{SM}}}\right)^{2}=\sin ^{2}(\beta-\alpha) .
$$

Representing the down-type (up-type) fermion final states by $\kappa_{D}\left(\kappa_{U}\right)$, the wrong-sign limit is defined by $\kappa_{D} \kappa_{V}<0$, that is, the down-type couplings have a minus relative sign to the SM couplings. Other wrong-sign limits could be defined but they are all excluded by experiment $[81,82]$. For completeness, the wrong sign limit is only possible in the type II model, and for the light Higgs scenario it implies $\alpha>0$, which leads to sizeable values of $\cos (\beta-\alpha)$.

On the left panel of Fig. 8 we present a plot of $\kappa_{D}$ as a function of $\kappa_{V}$, where all the theoretical and experimental constraints have been imposed, and the color code indicates the scale up to which the model is valid. Notice that the only region for which the model is valid to higher scales corresponds to $\kappa_{D}>0$ - thus the validity of the 2HDM up to high scales eliminates the wrong sign limit. To enforce this conclusion, consider the right plot in Fig. 8, wherein we show the values of $\cos (\beta-\alpha)$ as a function of the cutoff

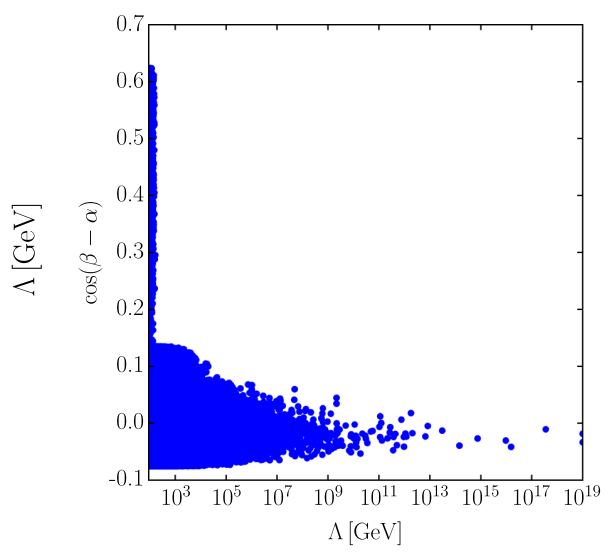

FIG. 8. Left: $\kappa_{D}$ as a function of $\kappa_{V}$ in the type II $2 \mathrm{HDM}$. The color bar indicates the cutoff scale at which the model is no longer valid. Right: $\cos (\beta-\alpha)$ as a function of the cutoff scale $\Lambda$. 

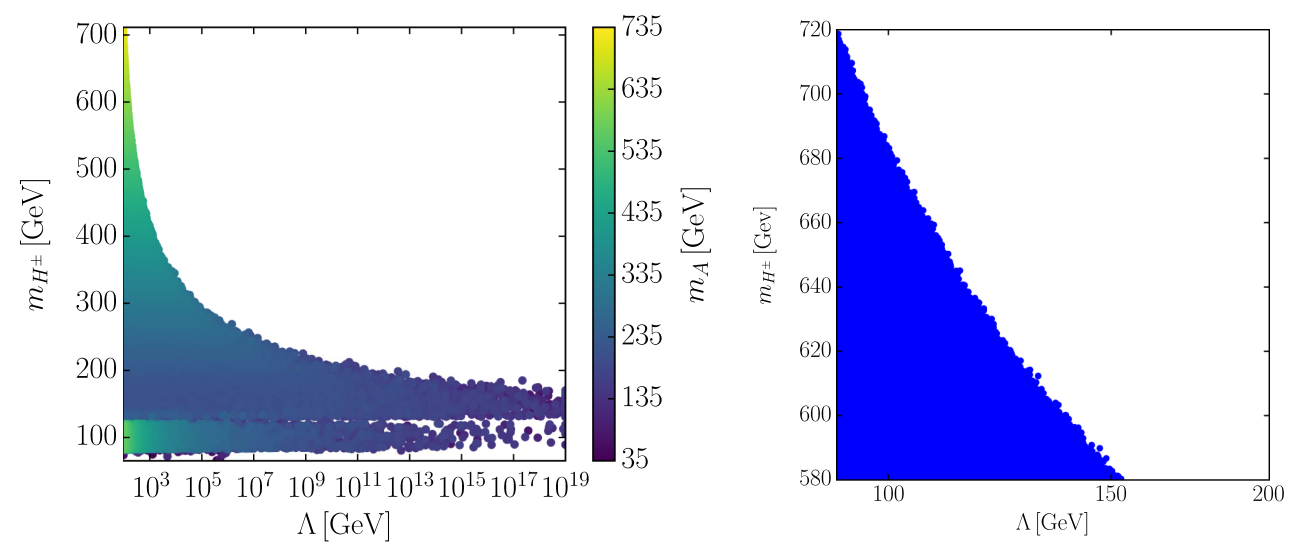

FIG. 9. Charged Higgs mass as a function of the cutoff scale $\Lambda$, for type I (left) and type II (right). The color coded bar in the left plot shows the value of $m_{A}$.

scale $\Lambda$. We clearly see that above about half a TeV the theory is valid only for points with very low values of $\cos (\beta-\alpha)$. Since the wrong sign limit can only occur with sizeable values of $\cos (\beta-\alpha)$ [81,82], it is therefore excluded if one requires the model to be valid up to scales as low as $1 \mathrm{TeV}$.

In fact, it is easy to understand why the wrong sign limit is excluded by the high scale behavior of type II, if one remembers the results from Fig. 3. Our analysis of those plots led us to conclude that validity up to high scales of type II placed us definitely in the decoupling regime-and as was shown in [82], the wrong sign limit corresponds to a nondecoupling regime, wherein the charged Higgs boson has an irreducible contribution to observables such as the diphoton width of the SM-like Higgs boson or the gluon fusion cross section.

\section{THE HEAVY HIGGS SCENARIO}

In this section, we will discuss the scenario where the heavier of the two $C P$-even Higgs bosons is the discovered $125 \mathrm{GeV}$ scalar [83]. In this scenario decoupling cannot happen because the lightest scalar mass is constrained to be below $125 \mathrm{GeV}$. Hence, one would expect that the theory would only be valid up to a certain scale, at least for the type II model - as we have seen, validity of type II to very high scales is only possible if the model is in the alignment limit and all extra scalar masses are heavy. Recall also that what makes possible the validity of the model up to the Planck scale is not only the fact that there is a new scalar, relative to the SM, but also that the mass scale is driven by the $M$ parameter and not by the quartic couplings. Since we are considering the possibility of scalars lighter than $125 \mathrm{GeV}$, the LEP constraints $[84,85]$ assume a special relevance in what follows.

In Fig. 9 we present the charged Higgs mass as a function of the cutoff scale for type I (left) and type II (right). In the type I model it can be clearly seen that there is a range of masses, both for the charged and pseudoscalar, that survive up to the Planck scale. In particular, acceptable charged masses are above the LEP bound but below $200 \mathrm{GeV}$. Therefore, the type I model survives up to the Planck scale if the charged Higgs boson and/or the pseudoscalar are light. On the other hand, in type II, the stringent bound of $580 \mathrm{GeV}$ not only precludes the possibility of a light charged scalar but the theory ceases to be valid already at $\Lambda \approx 150 \mathrm{GeV}$. Even if we consider only the appearance of Landau poles the type II model is valid only up to about $1.5 \mathrm{TeV}$.

In fact, in type I, it is not only the charged Higgs boson that needs to be light for the model to be valid up to the Planck scale. In Fig. 10 we show $m_{A}-m_{H^{ \pm}}$vs $m_{h}-m_{H^{ \pm}}$ for type I (left) and type II (right). The color code shows at which energy either a Landau pole occurs or one of the theoretical conditions is violated. It is clear that for type I to be valid up to the Planck scale the pseudoscalar also needs to be light, and in fact all mass differences have to be below $100 \mathrm{GeV}$. We have also checked that the value of $M$ has to be of the same order and lies between $40 \mathrm{GeV}$ and $120 \mathrm{GeV}$. The cutoff scale has no major influence on the range ${ }^{7}$ of $\sin (\beta-\alpha)$ nor on the range of $\tan \beta$. In fact, there is only a slight reduction in the allowed region with a slight increase in the lower bound of $\tan \beta$, which moves closer to $\tan \beta>4$.

The situation is radically different in the type II model, as can be appreciated from the plot on the right in Fig. 10. We may conclude from that plot that, due to the bound on the charged Higgs mass, the type II model barely survives up to a scale of $200 \mathrm{GeV}$. Once again, this is due to the fact that validity of the type II model up to high scales eliminates the possibility of nondecoupling regimes, of which the heavy Higgs scenario is certainly one. However, there are regions in the parameter space of type I, in the heavy Higgs scenario, valid up to the Planck scale, which is

\footnotetext{
${ }^{7}$ Note that in the heavy Higgs scenario the alignment limit is attained for $\sin (\beta-\alpha) \approx 0$.
} 

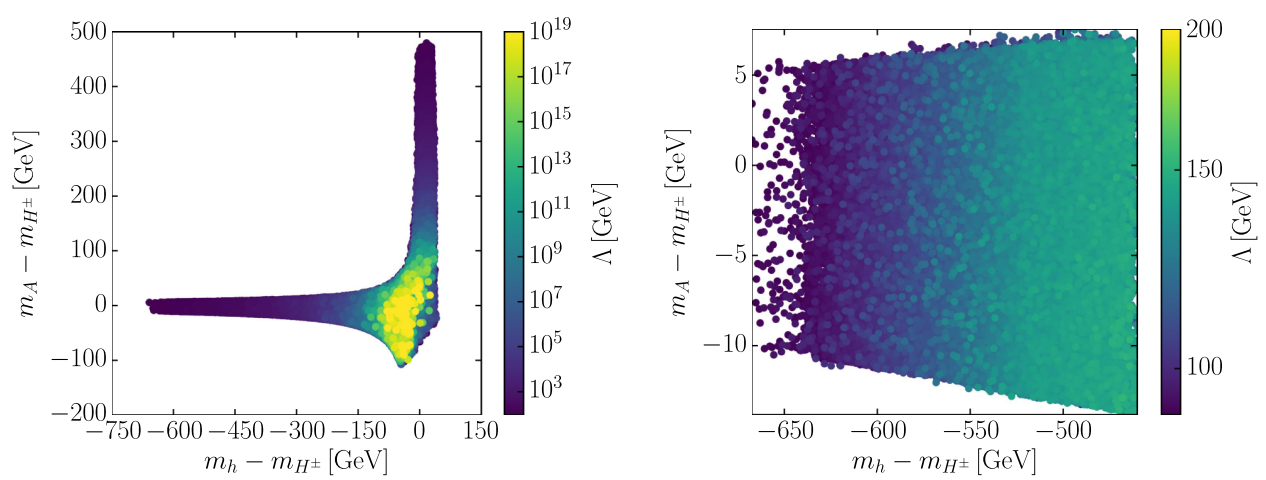

FIG. 10. The mass difference $m_{A}-m_{H^{ \pm}}$vs $m_{h}-m_{H^{ \pm}}$for type I (left) and type II (right). The color code shows the cutoff scale $\Lambda$.

certainly surprising. The conditions for this to happen are $m_{H^{ \pm}} \approx m_{A} \approx M$ and all masses below about $200 \mathrm{GeV}$. There is no preferred value of the lightest scalar mass even when one requires the model to be valid up to the Planck scale. ${ }^{8}$ And as in the case of the light Higgs scenario, the running does not force the type I model to move further close to the alignment limit.

\section{HIGH SCALE BEHAVIOR AND 2HDM SYMMETRIES}

The potential presented in Eq. (2.1) is $\mathbb{Z}_{2}$ symmetric, softly broken by the $m_{12}^{2}$ (or $M$ ) term. The potential has an exact $\mathbb{Z}_{2}$ symmetry when $M=0$. We have shown in Fig. 2 (left) $M$ as a function of the cutoff scale $\Lambda$ in the type II 2HDM while in Fig. 6 we show the same plot but now for type I. These plots allow us to analyze the possibility of the $\mathbb{Z}_{2}$ symmetry actually being an exact symmetry, unbroken even softly. To do that, we simply need to investigate the possibility of $M$ - and therefore $m_{12}^{2}$ - being equal to zero while the $2 \mathrm{HDM}$ is still valid up to high-energy scales.

Now, it is quite clear from Fig. 2 (left) that $M=0$ is only a possibility for type II if the validity scale of the model is well below $1 \mathrm{TeV}^{9}$ - thus one can conclude that a type II model with an exact $\mathbb{Z}_{2}$ symmetry is already strongly ruled out. For the type I model, Fig. 6 shows that $M=0$ is a possibility for theories valid up to scales of roughly $10 \mathrm{TeV}$, but no more than that. Thus an exact $\mathbb{Z}_{2}$ symmetry is a possibility for a type I model, but only if new physics is present at $\sim 10 \mathrm{TeV}$. Once again, the explanation for this is due to the fact that most nondecoupling regimes are excluded if one considers the $2 \mathrm{HDM}$ valid to very high energies-and the motivation for introducing a soft breaking term in the potential is indeed to allow for the

\footnotetext{
${ }^{8}$ It is easier to fit the existing data with $m_{h}$ above roughly $62.5 \mathrm{GeV}$, but that is due to possible $H \rightarrow h$ h decays potentially enlarging the branching ratio of $H$ beyond what is acceptable to be compatible with the LHC data on the Higgs rates.

${ }^{9}$ We differ from previous calculations [29,31,32] due to the higher charged Higgs mass bound, which had used a value of $350 \mathrm{GeV}$.
}

possibility of a decoupling regime occurring. Still, as we have showed for type I, although there is no decoupling in the heavy Higgs scenario, it is only the existence of the term $m_{12}^{2}$ that allows the model to survive up to the Planck scale. With $m_{12}^{2}$ absent, the 2HDM becomes a nondecoupled theory, where some quantities (such as the diphoton width) never conform to SM expectations [44].

We were also able to investigate the possibility of the 2HDM possessing a $U(1)$ Peccei-Quinn symmetry [86]. This symmetry would imply $\lambda_{5}=0$ and in its exact form, also $m_{12}^{2}=0$. Again one can break it with a soft breaking term and the symmetry is extended to the Yukawa sector in the same manner as the $\mathbb{Z}_{2}$ model. But in this model the pseudoscalar $A$ is massless if no soft breaking term $m_{12}^{2}$ is introduced, so we are not interested in the exact symmetry scenario. And since $\lambda_{5}=0$ is enforced by a symmetry, it is a fixed point in the RGE running of the quartic couplings. Thus we may ask if, given all collider constraints existent, and requiring the model to be valid up to very high scales, the RGE running is making the $\mathbb{Z}_{2}$ model tending to the Peccei-Quinn one. This would happen if only values of $\lambda_{5}$ close to zero-more generically, of magnitude much smaller than the remaining couplings-would survive the running. However, the results show that all $\lambda_{i}$ have similar allowed ranges at the Planck scale and therefore $\lambda_{5}$ does not approach zero faster than any of the other $\lambda_{i}$. Moreover, at the Planck scale the range of allowed values of $\lambda_{5}$ is similar to all other $\lambda_{i}$. This was checked for all scenarios presented in this work.

\section{TWO-LOOP RUNNING, ONE-LOOP MATCHING}

The two-loop $\beta$ functions of the 2HDM are well established in the literature (see, for instance, the implementation of these functions in SARAH [87-91], and the explicit expressions in [32]), and it has been shown that their impact on 2HDM phenomenology may not be negligible $[32,35,92]$ for certain regions of parameter space. Therefore, the question may arise, how would the results we have hitherto presented change, if one considers 
two-loop running of the parameters? The answer is, not very much. Before presenting the results of such running, some general considerations are in order:

(i) In this paper, we have been concerned with general trends in the 2HDM parameter space, not precision studies. The use of higher-order expressions will surely change predictions for cutoff scales or mass bounds for specific parameter choices, but the overall conclusions, we will show, remain unchanged.

(ii) As has been discussed previously, one of the greatest contributors in reducing the allowed 2HDM parameter space is the development of Landau poles at high scales. This implies that the theory becomes nonperturbative at high scales-for such parameter choices, care must be exercised when interpreting higher-order corrections. If the model is not perturbative already when one is considering one-loop expressions, introducing two-loop ones might be problematic or useless.

(iii) Running with an $N$-loop $\beta$ function requires that one obtains the parameters of the model (Yukawa, quartic and gauge couplings, for instance) through $N-1$-loop expressions for the masses of the model's particles (the so-called "matching" procedure ${ }^{10}$ ). One-loop expressions for quark, gauge boson $[93,94]$ and scalar masses (see, for instance, [95]) were therefore considered.

(iv) One-loop expressions for the unitarity conditions $[35,96]$ were also considered at all scales.

(v) It is well known that one-loop corrections to the quartic couplings can be very large in some regions of the 2HDM parameter space [97,98]. As these are then used in the matching procedure, large deviations are to be expected in the one-loop matching procedure $[99,100]$ compared to the values obtained via treelevel matching. Therefore, substantial phenomenological differences in individual choices of parameters may be expected, but again, an overall analysis of the parameter space will yield mostly the same trends in terms of scale validity and mass bounds.

We therefore repeated the analysis of the several scenarii of the previous sections, but now with two-loop $\beta$ functions, one-loop mass matching and one-loop unitarity conditions. In Fig. 11 we show the analogue of the left plot in Fig. 5 for the validity analysis of the Type I model including present collider constraints, redone with the higher-order analysis described above. It is plain to see, comparing both figures, that the conclusions drawn in Sec. V B remain unchanged-there are regions of parameter space for which the type I model is valid all the way up to the Planck scale; and if the charged Higgs mass is above roughly $500 \mathrm{GeV}$, the model can only survive to high scales

\footnotetext{
${ }^{10} \mathrm{~A}$ recent work, however, proposed that $N$-loop matching should be considered [92].
}

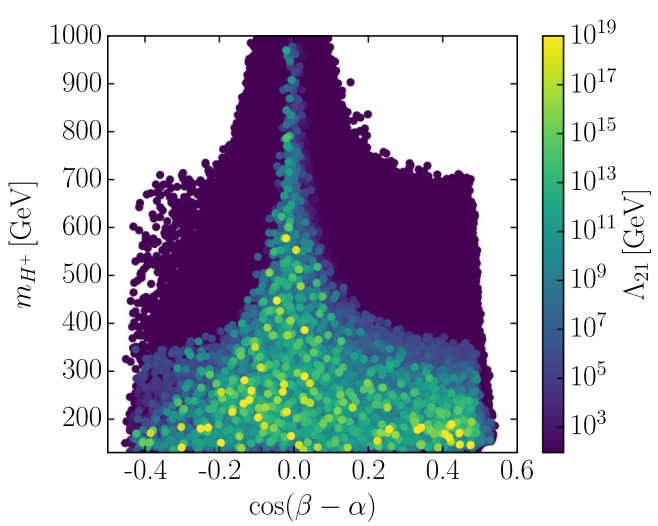

FIG. 11. Charged Higgs mass vs $\cos (\beta-\alpha)$ in the type I 2HDM, with two-loop running, one-loop matching and one-loop unitarity conditions. The color bar shows the cutoff scale.

if it is aligned. We present this plot as an example of the results obtained with the higher-order procedure-the conclusions drawn in the previous sections, with minor changes, remain valid. The one major difference between the plots in Fig. 11 and 5 is how much "sharper" the narrow, aligned-like parameter space in the former is, compared to the latter. This can be tracked to the effect of the one-loop unitarity conditions on the parameter space considered. Going through the different 2HDM versions analyzed, one concludes that:

(i) For the light Higgs scenario in type I, the model is valid all the way up to the Planck scale if alignment occurs; the mass differences between the different models behave, for the higher-order analysis, just as in Fig. 5; smaller values of the $M$ coefficient now survive RG running up to a scale of $\sim 10^{7} \mathrm{GeV}$, instead of up to $10^{5} \mathrm{GeV}$ as in Fig. 6 .

(ii) For the heavy Higgs case in type I, the two-loop running, one-loop matching procedure does not change the fact that there are $2 \mathrm{HDM}$ parameter space regions for which this scenario is valid all the way up to the Planck scale, and, as in Fig. 9, such validity occurs with all extra scalars with masses below roughly $200 \mathrm{GeV}$.

(iii) For the light Higgs scenario in type II, as before, requiring that the model be valid up to a scale as low as $1 \mathrm{TeV}$ already implies a strongly aligned regime; and as before, there are parameter regions of the model for which it is valid all the way up to the Planck scale, provided all extra scalar masses are close in value, and the two-loop running, one-loop matching procedure yields results akin to those of Fig. 3.

(iv) Finally, the heavy Higgs scenario in type II is completely excluded with the higher-order procedure. Again, the large contributions of one-loop corrections to the quartic couplings that are used in the matching procedure lead to an exclusion of the 
parameter space based on perturbative unitarity considerations already at the weak scale. The same is true for the wrong sign scenario in the light Higgs scenario in type II.

Therefore, the one substantial change from the one-loop running, tree-level analysis is the exclusion of the heavy Higgs scenario in type II already at the weak scale. However, we could argue that this is not surprising-in the previous analysis, this scenario was already excluded at the few $\mathrm{TeV}$ scale, which hinted that a very careful selection of parameters needed to be made if one were to consider a minimally successful implementation of the model. The one-loop matching and unitarity analysis further constrains the validity of the scenario, seemingly excluding it - although the existence of some corner of parameter space where it could be viably implemented cannot be wholly excluded and could be the objective of a dedicated study beyond the scope of the current paper.

The conclusion therefore is that the two-loop running, one-loop matching procedure does not add much to the conclusions drawn from the simpler analysis-clearly, if one is aiming at precision studies, higher-order contributions are fundamental, but a generic analysis of the 2HDM's validity can already be obtained with one-loop running, tree-level matching. And where substantial differences occur, they are related to large values of the quartic couplings, wherein the 2HDM's perturbativity is already questionable, even at tree level. On that note, one could argue that one-loop running is even a more adequate approach-in Ref. [101] the running of the top Yukawa coupling was considered in supersymmetric (SUSY) models, up to four loops, to analyze the available parameter space for the so-called "quasi-infra-red fixed point". As in the current work, the possibility of Landau poles occurring curtailed the available parameter space. What one observes from Fig. 2 of that work is an "oscillating" behavior, in which $\beta$ functions up to odd orders develop Landau poles for smaller values of low-scale couplings, whereas evenorder $\beta$ functions allow for a larger domain of attraction at the weak scale. Assuming an asymptotic nature for the perturbation theory [102] of these $\beta$ functions, a Padé-Borel resummation was then undertaken. Fig. 3 of Ref. [101] then shows that, for larger values of the couplings, the one-loop $\beta$ function seems a better approximation to the resummed theory than the four-loop one (again, for emphasis, for the region of larger couplings). Extrapolating this argument from SUSY to the 2HDM situation herein discussed, one could argue that, if already at tree level, or one-loop, one is running into issues of perturbative breakdown due to large values of couplings, considering higher-order contributions in such situations may be of dubious usefulness.

\section{CONCLUSIONS}

We have analyzed the high-scale behavior of a softly broken $\mathbb{Z}_{2}$ symmetric 2 HDM focusing on two particular
Yukawa types, type I and type II. If the lightest $C P$-even scalar is the $125 \mathrm{GeV}$ one, there are regions of the parameter space for both types that survive up to the Planck scale. This is a confirmation of many previous studies in the literature. There are, however, new and quite interesting results, some of them unexpected that we will now discuss.

One of the most interesting conclusions of our study is that for the model to be close to the alignment limit it is enough to require it to be valid up to about $1 \mathrm{TeV}$ and at the same time to have one of the scalar masses above about $500 \mathrm{GeV}$ (in the specific case of type II $B$-physics bounds force the charged Higgs mass to be above $580 \mathrm{GeV}$ ). No other bounds need to be considered to reach this limit. As the scale up to which we want the model to be valid increases, the allowed region of parameter space moves closer and closer to alignment. Therefore, alignment is reached via decoupling — at least a large (above roughly $500 \mathrm{GeV}$ ) scalar mass is required.

On the contrary, we have shown that for type I, for which there are no strong bounds on the scalar masses, validity up to the Planck scale will not imply alignment, if the masses are low enough. In fact, even when all experimental constraints are considered, the type I model can be far from alignment, except for large scalar masses where we then recover the results obtained for type II. In this sense, validity up to higher scales (as low as $1 \mathrm{TeV}$ in certain cases), complemented with one sufficiently large scalar mass (above $500 \mathrm{GeV}$ ) implies alignment in the 2HDM, a phenomenon we might call "radiative alignment." In this sense, alignment in the 2HDM is therefore fundamentally caused by the behavior of the theory at high scales, instead as, for instance, the occurrence of symmetries - of which the inert doublet model [103-106] is a prime example; another possibility would be the model developed in Ref. [107].

The validity up to high scales of the scenario where the heaviest $C P$-even scalar is the $125 \mathrm{GeV}$ Higgs was analyzed here for the first time. Interestingly, we have shown that there are regions of the parameter space where a type I model, in the heavy Higgs scenario, is valid up to the Planck scale. That is, a model with no decoupling limit can be valid up to the Planck scale. The most interesting point to note is that also in this case it is the soft parameter $M$ that sets the mass scale for validity at high energies. In fact, all masses have to be below about $200 \mathrm{GeV}$ to ensure that the model does not require new physics up to the Planck scale. On the contrary, and again due to the bound on the charged Higgs mass, the type II model in the heavy Higgs scenario does not survive, even to a scale of just a few hundred GeV. In all these scenarios we should highlight the important role played by the parameter $M$. Indeed, because the quartic couplings become increasingly small, all models that survive up to the Planck scale need a nonzero $M$ and all masses are of the order of $M$ (except for the $125 \mathrm{GeV}$ Higgs boson).

The previous result is even more interesting when combined with knowledge that nondecoupling scenarios 
in type II, such as the wrong sign limit, will not survive to scales as low as a few TeV. As the quartic couplings increase with energy, they have to be quite small to survive the running. Hence, any nondecoupling regime that needs large quartic couplings will not survive to high scales.

We have also shown that the model does not approach the exact $\mathbb{Z}_{2}$ symmetry nor the softly broken $U(1)$ symmetry when the validity of the theory is required up to the Planck scale. In fact, the value of $M^{2}=0$ is disallowed for type II already at a scale well below $1 \mathrm{TeV}$ while for type I it happens at a scale of about $10 \mathrm{TeV}$. As for the softly broken $U(1)$ where $\lambda_{5}=0$, we have shown that requiring the validity of the model up to the Planck scale forces all the quartic couplings to be small so that $\lambda_{5}$ behaves just like the other quartic couplings.

Finally, repeating the analysis with running from twoloop $\beta$ functions, one-loop matching (and one-loop unitarity conditions) does not produce significantly different results.

\section{ACKNOWLEDGMENTS}

We would like to thank Jonas Wittbrodt for useful discussions and for providing the 2HDM samples. P. B. acknowledges the support by the DFG-funded Doctoral School Karlsruhe School of Elementary and Astroparticle Physics: Science and Technology. M. M. acknowledges financial support from the DFG project "Precision Calculations in the Higgs Sector-Paving the Way to the New Physics Landscape" (No. MU 3138/1-1). P. F. and R. S. are supported in part by the National Science Centre, Poland, the HARMONIA project under Contract No. UMO-2015/18/M/ST2/00518.

\section{APPENDIX: RGES FOR THE 2HDM}

The one-loop RGEs for the gauge couplings, Yukawa couplings and $\lambda$ 's are taken from [108]. The one-loop RGEs for the quadratic parameters $m_{i j}^{2}$ from Eq. (2.1) are taken from [109]. We define

$$
\beta_{x}=16 \pi^{2} \frac{\partial x}{\partial \ln \mu} .
$$

The RGEs for the $U(1)_{Y}, S U(2)_{L}$ and $S U(3)$ gauge couplings $g, g^{\prime}$ and $g_{s}$, respectively, are given as

$$
\begin{aligned}
& \beta_{g_{s}}=-7 g_{s}^{3}, \\
& \beta_{g}=-3 g^{3}, \\
& \beta_{g^{\prime}}=7 g^{\prime 3} .
\end{aligned}
$$

For the Yukawa sector (see below Eqs. (A38),(A39),(A40) for the definition of the Yukawa matrices $Y_{x}$ ) we have in type I

$$
\begin{gathered}
\beta_{Y_{u}}=a_{u} Y_{u}+T_{22} Y_{u}-\frac{3}{2}\left(Y_{d} Y_{d}^{\dagger}-Y_{u} Y_{u}^{\dagger}\right) Y_{u}, \\
\beta_{Y_{d}}=a_{d} Y_{d}+T_{22} Y_{d}+\frac{3}{2}\left(Y_{d} Y_{d}^{\dagger}-Y_{u} Y_{u}^{\dagger}\right) Y_{d}, \\
\beta_{Y_{e}}=a_{e} Y_{e}+T_{22} Y_{e}+\frac{3}{2} Y_{e} Y_{e}^{\dagger} Y_{e},
\end{gathered}
$$

and in type II

$$
\begin{gathered}
\beta_{Y_{u}}=a_{u} Y_{u}+T_{22} Y_{u}+\frac{1}{2}\left(Y_{d} Y_{d}^{\dagger}+3 Y_{u} Y_{u}^{\dagger}\right) Y_{u}, \\
\beta_{Y_{d}}=a_{d} Y_{d}+T_{11} Y_{d}+\frac{1}{2}\left(Y_{u} Y_{u}^{\dagger}+3 Y_{d} Y_{d}^{\dagger}\right) Y_{d}, \\
\beta_{Y_{e}}=a_{e} Y_{e}+T_{11} Y_{e}+\frac{3}{2} Y_{e} Y_{e}^{\dagger} Y_{e},
\end{gathered}
$$

with

$$
\begin{gathered}
a_{d}=-8 g_{s}^{2}-\frac{9}{4} g^{2}-\frac{5}{12} g^{\prime 2}, \\
a_{u}=-8 g_{s}^{2}-\frac{9}{4} g^{2}-\frac{17}{12} g^{\prime 2}, \\
a_{e}=-\frac{9}{4} g^{2}-\frac{15}{4} g^{\prime 2} .
\end{gathered}
$$

For type I, we define

$$
\begin{gathered}
T_{11}=0, \\
T_{22}=3 Y_{u}^{\dagger} Y_{u}+3 Y_{d}^{\dagger} Y_{d}+Y_{e}^{\dagger} Y_{e},
\end{gathered}
$$

and for type II, we have

$$
\begin{gathered}
T_{11}=3 Y_{d}^{\dagger} Y_{d}+Y_{e}^{\dagger} Y_{e}, \\
T_{22}=3 Y_{u}^{\dagger} Y_{u} .
\end{gathered}
$$

For the quartic couplings, we have in type I

$$
\begin{aligned}
\beta_{\lambda_{1}}= & 12 \lambda_{1}^{2}+4 \lambda_{3}^{2}+4 \lambda_{3} \lambda_{4}+2 \lambda_{4}^{2}+2 \lambda_{5}^{2}+\frac{9}{4} g^{4} \\
& +\frac{3}{2} g^{2} g^{\prime 2}+\frac{3}{4} g^{\prime 4}-4 \gamma_{1} \lambda_{1}, \\
\beta_{\lambda_{2}}= & 12 \lambda_{2}^{2}+4 \lambda_{3}^{2}+4 \lambda_{3} \lambda_{4}+2 \lambda_{4}^{2}+2 \lambda_{5}^{2}+\frac{9}{4} g^{4}+\frac{3}{2} g^{2} g^{\prime 2} \\
& +\frac{3}{4} g^{\prime 4}-4 \gamma_{2} \lambda_{2}-12 \operatorname{Tr}\left[Y_{d}^{\dagger} Y_{d} Y_{d}^{\dagger} Y_{d}+Y_{u}^{\dagger} Y_{u} Y_{u}^{\dagger} Y_{u}\right] \\
& -4 \operatorname{Tr}\left[Y_{e}^{\dagger} Y_{e} Y_{e}^{\dagger} Y_{e}\right],
\end{aligned}
$$




$$
\begin{gathered}
\beta_{\lambda_{3}=}\left(\lambda_{1}+\lambda_{2}\right)\left(6 \lambda_{3}+2 \lambda_{4}\right)+4 \lambda_{3}^{2}+2 \lambda_{4}^{2}+2 \lambda_{5}^{2} \\
+\frac{9}{4} g^{4}-\frac{3}{2} g^{2} g^{2}+\frac{3}{4} g^{\prime 4}-2\left(\gamma_{1}+\gamma_{2}\right) \lambda_{3}, \\
\beta_{\lambda_{4}}=2\left(\lambda_{1}+\lambda_{2}\right) \lambda_{4}+8 \lambda_{3} \lambda_{4}+4 \lambda_{4}^{2}+8 \lambda_{5}^{2} \\
\quad-2\left(\gamma_{1}+\gamma_{2}\right) \lambda_{4}+3 g^{2} g^{\prime 2}, \\
\beta_{\lambda_{5}}=2\left(\lambda_{1}+\lambda_{2}+4 \lambda_{3}+6 \lambda_{4}\right) \lambda_{5}-2\left(\gamma_{1}+\gamma_{2}\right) \lambda_{5},
\end{gathered}
$$

and in type II

$$
\begin{aligned}
\beta_{\lambda_{1}}= & 12 \lambda_{1}^{2}+4 \lambda_{3}^{2}+4 \lambda_{3} \lambda_{4}+2 \lambda_{4}^{2}+2 \lambda_{5}^{2}+\frac{9}{4} g^{4}+\frac{3}{2} g^{2} g^{\prime 2} \\
& +\frac{3}{4} g^{\prime 4}-4 \gamma_{1} \lambda_{1}-12 \operatorname{Tr}\left[Y_{d}^{\dagger} Y_{d} Y_{d}^{\dagger} Y_{d}\right]-4 \operatorname{Tr}\left[Y_{e}^{\dagger} Y_{e} Y_{e}^{\dagger} Y_{e}\right],
\end{aligned}
$$

$$
\begin{aligned}
\beta_{\lambda_{2}=} & 12 \lambda_{2}^{2}+4 \lambda_{3}^{2}+4 \lambda_{3} \lambda_{4}+2 \lambda_{4}^{2}+2 \lambda_{5}^{2}+\frac{9}{4} g^{4}+\frac{3}{2} g^{2} g^{\prime 2} \\
& +\frac{3}{4} g^{4}-4 \gamma_{2} \lambda_{2}-12 \operatorname{Tr}\left[Y_{u}^{\dagger} Y_{u} Y_{u}^{\dagger} Y_{u}\right], \\
\beta_{\lambda_{3}=} & \left(\lambda_{1}+\lambda_{2}\right)\left(6 \lambda_{3}+2 \lambda_{4}\right)+4 \lambda_{3}^{2}+2 \lambda_{4}^{2}+2 \lambda_{5}^{2}+\frac{9}{4} g^{4} \\
& -\frac{3}{2} g^{2} g^{\prime 2}+\frac{3}{4} g^{\prime 4}-2\left(\gamma_{1}+\gamma_{2}\right) \lambda_{3}-12 \operatorname{Tr}\left[Y_{d}^{\dagger} Y_{d} Y_{u}^{\dagger} Y_{u}\right],
\end{aligned}
$$

$\beta_{\lambda_{4}}=2\left(\lambda_{1}+\lambda_{2}\right) \lambda_{4}+8 \lambda_{3} \lambda_{4}+4 \lambda_{4}^{2}+8 \lambda_{5}^{2}-2\left(\gamma_{1}+\gamma_{2}\right) \lambda_{4}$

$$
+3 g^{2} g^{\prime 2}+12 \operatorname{Tr}\left[Y_{d}^{\dagger} Y_{d} Y_{u}^{\dagger} Y_{u}\right]
$$

$\beta_{\lambda_{5}}=2\left(\lambda_{1}+\lambda_{2}+4 \lambda_{3}+6 \lambda_{4}\right) \lambda_{5}-2\left(\gamma_{1}+\gamma_{2}\right) \lambda_{5}$,

with

$$
\begin{aligned}
& \gamma_{1}=\frac{9}{4} g^{2}+\frac{3}{4} g^{\prime 2}-T_{11}, \\
& \gamma_{2}=\frac{9}{4} g^{2}+\frac{3}{4} g^{\prime 2}-T_{22} .
\end{aligned}
$$

For the dimensionful couplings we have

$$
\begin{gathered}
\beta_{m_{11}^{2}}=6 \lambda_{1} m_{11}^{2}+\left(4 \lambda_{3}+2 \lambda_{4}\right) m_{22}^{2}-2 \gamma_{1} m_{11}^{2}, \\
\beta_{m_{22}^{2}}=\left(4 \lambda_{3}+2 \lambda_{4}\right) m_{11}^{2}+6 \lambda_{2} m_{22}^{2}-2 \gamma_{2} m_{22}^{2}, \\
\beta_{m_{12}^{2}}=\left(2 \lambda_{3}+4 \lambda_{4}+6 \lambda_{5}\right) m_{12}^{2}-\left(\gamma_{1}+\gamma_{2}\right) m_{12}^{2} .
\end{gathered}
$$

The RGEs for the VEVs are given by $[110,111]$

$$
\beta_{v_{1}}=\gamma_{1} v_{1}
$$

$$
\beta_{v_{2}}=\gamma_{2} v_{2}
$$

Our starting values are given by

$$
\begin{aligned}
& g_{s}=\sqrt{4 \pi \alpha_{s}}, \\
& g=\frac{2 m_{W}}{v}, \\
& g^{\prime}=2 \frac{\sqrt{m_{Z}^{2}-m_{W}^{2}}}{v}, \\
& Y_{u}=\frac{\sqrt{2}}{v_{2}}\left(\begin{array}{ccc}
m_{u} & 0 & 0 \\
0 & m_{c} & 0 \\
0 & 0 & m_{t}
\end{array}\right), \\
& Y_{d}=\frac{\sqrt{2}}{v_{d}} V_{\mathrm{CKM}}\left(\begin{array}{ccc}
m_{d} & 0 & 0 \\
0 & m_{s} & 0 \\
0 & 0 & m_{b}
\end{array}\right) V_{\mathrm{CKM}}^{\dagger} \text {, } \\
& Y_{e}=\frac{\sqrt{2}}{v_{e}}\left(\begin{array}{ccc}
m_{e} & 0 & 0 \\
0 & m_{\mu} & 0 \\
0 & 0 & m_{\tau}
\end{array}\right) \text {, } \\
& V_{\mathrm{CKM}}=1_{3 \times 3},
\end{aligned}
$$

where $\alpha_{s}=g_{s}^{2} /(4 \pi)$ is the strong coupling constant. In type I, we have

$$
\begin{aligned}
& v_{e}=v_{2}, \\
& v_{d}=v_{2},
\end{aligned}
$$

and in type II,

$$
\begin{aligned}
& v_{e}=v_{1}, \\
& v_{d}=v_{1} .
\end{aligned}
$$

The fermion masses are chosen as [112-115]

$$
\begin{gathered}
m_{u}=0.1 \mathrm{GeV}, \\
m_{c}=1.51 \mathrm{GeV}, \\
m_{t}=172.5 \mathrm{GeV}, \\
m_{d}=0.1 \mathrm{GeV}, \\
m_{s}=0.1 \mathrm{GeV}, \\
m_{b}=4.92 \mathrm{GeV},
\end{gathered}
$$




$$
\begin{gathered}
m_{e}=0.510998928 \times 10^{-3} \mathrm{GeV}, \\
m_{\mu}=0.1056583715 \mathrm{GeV}, \\
m_{\tau}=1.77682 \mathrm{GeV} .
\end{gathered}
$$

The VEV is given by

$$
\begin{gathered}
G_{F}=1.1663787 \times 10^{-5} \mathrm{GeV}^{-2}, \\
v=\frac{1}{\sqrt{\sqrt{2} G_{F}}},
\end{gathered}
$$

and the strong coupling is

$$
\alpha_{s}=0.119
$$

The $\mathrm{W}$ and $\mathrm{Z}$ boson masses are given by $[112,113]$

$$
\begin{aligned}
& m_{W}=80.385 \mathrm{GeV}, \\
& m_{Z}=91.1876 \mathrm{GeV} .
\end{aligned}
$$

[1] G. Aad et al. (ATLAS Collaboration), Phys. Lett. B 716, 1 (2012).

[2] S. Chatrchyan et al. (CMS Collaboration), Phys. Lett. B 716, 30 (2012).

[3] T. D. Lee, Phys. Rev. D 8, 1226 (1973).

[4] P. Q. Hung, Phys. Rev. Lett. 42, 873 (1979).

[5] N. Cabibbo, L. Maiani, G. Parisi, and R. Petronzio, Nucl. Phys. B158, 295 (1979).

[6] R. A. Flores and M. Sher, Phys. Rev. D 27, 1679 (1983).

[7] M. Lindner, Z. Phys. C 31, 295 (1986).

[8] M. Sher, Phys. Rep. 179, 273 (1989).

[9] M. Lindner, M. Sher, and H. W. Zaglauer, Phys. Lett. B 228, 139 (1989).

[10] C. Ford, D. R. T. Jones, P. W. Stephenson, and M. B. Einhorn, Nucl. Phys. B395, 17 (1993).

[11] M. Sher, Phys. Lett. B 317, 159 (1993); 331, 448 (1994).

[12] G. Isidori, G. Ridolfi, and A. Strumia, Nucl. Phys. B609, 387 (2001).

[13] M. B. Einhorn and D. R. T. Jones, J. High Energy Phys. 04 (2007) 051.

[14] F. Bezrukov, M. Y. Kalmykov, B. A. Kniehl, and M. Shaposhnikov, J. High Energy Phys. 10 (2012) 140.

[15] G. Degrassi, S. Di Vita, J. Elias-Miró, J. R. Espinosa, G. F. Giudice, G. Isidori, and A. Strumia, J. High Energy Phys. 08 (2012) 098.

[16] D. Buttazzo, G. Degrassi, P. P. Giardino, G. F. Giudice, F. Sala, A. Salvio, and A. Strumia et al., J. High Energy Phys. 12 (2013) 089.

[17] O. Lebedev, Eur. Phys. J. C 72, 2058 (2012).

[18] J. Elias-Miro, J. R. Espinosa, G. F. Giudice, H. M. Lee, and A. Strumia, J. High Energy Phys. 06 (2012) 031.

[19] G. M. Pruna and T. Robens, Phys. Rev. D 88, 115012 (2013).

[20] R. Costa, A. P. Morais, M. O. P. Sampaio, and R. Santos, Phys. Rev. D 92, 025024 (2015).

[21] V. Branchina and E. Messina, Phys. Rev. Lett. 111, 241801 (2013).

[22] V. Branchina, E. Messina, and A. Platania, J. High Energy Phys. 09 (2014) 182.
[23] V. Branchina, E. Messina, and M. Sher, Phys. Rev. D 91, 013003 (2015).

[24] V. Branchina, E. Messina, and D. Zappala, Europhys. Lett. 116, 21001 (2016).

[25] G. Kreyerhoff and R. Rodenberg, Phys. Lett. B 226, 323 (1989).

[26] S. Nie and M. Sher, Phys. Lett. B 449, 89 (1999).

[27] S. Kanemura, T. Kasai, and Y. Okada, Phys. Lett. B 471, 182 (1999).

[28] P. M. Ferreira and D. R. T. Jones, J. High Energy Phys. 08 (2009) 069.

[29] N. Chakrabarty, U. K. Dey, and B. Mukhopadhyaya, J. High Energy Phys. 12 (2014) 166.

[30] P. S. B. Dev and A. Pilaftsis, J. High Energy Phys. 12 (2014) 024; 11 (2015) 147(E).

[31] D. Das and I. Saha, Phys. Rev. D 91, 095024 (2015).

[32] D. Chowdhury and O. Eberhardt, J. High Energy Phys. 11 (2015) 052.

[33] P. Ferreira, H. E. Haber, and E. Santos, Phys. Rev. D 92, 033003 (2015); 94, 059903(E) (2016).

[34] N. Chakrabarty and B. Mukhopadhyaya, Eur. Phys. J. C 77, 153 (2017).

[35] V. Cacchio, D. Chowdhury, O. Eberhardt, and C. W. Murphy, J. High Energy Phys. 11 (2016) 026.

[36] N. Chakrabarty and B. Mukhopadhyaya, Phys. Rev. D 96, 035028 (2017).

[37] S. Gori, H. E. Haber, and E. Santos, J. High Energy Phys. 06 (2017) 110.

[38] P. M. Ferreira and B. Swiezewska, J. High Energy Phys. 04 (2016) 099.

[39] A. L. Cherchiglia and C. C. Nishi, J. High Energy Phys. 11 (2017) 106.

[40] P. M. Ferreira, R. Santos, and A. Barroso, Phys. Lett. B 603, 219 (2004); 629, 114(E) (2005).

[41] S. L. Glashow and S. Weinberg, Phys. Rev. D 15, 1958 (1977).

[42] E. A. Paschos, Phys. Rev. D 15, 1966 (1977).

[43] V. D. Barger, J. L. Hewett, and R. J. N. Phillips, Phys. Rev. D 41, 3421 (1990). 
[44] S. Kanemura, Y. Okada, E. Senaha, and C. P. Yuan, Phys. Rev. D 70, 115002 (2004).

[45] S. Davidson and H. E. Haber, Phys. Rev. D 72, 035004 (2005); 72, 099902(E) (2005).

[46] J. F. Gunion and H. E. Haber, Phys. Rev. D 67, 075019 (2003).

[47] N. G. Deshpande and E. Ma, Phys. Rev. D 18, 2574 (1978).

[48] K. G. Klimenko, Teor. Mat. Fiz. 62, 87 (1985) [Theor. Math. Phys. 62, 58 (1985)].

[49] I. P. Ivanov, Phys. Rev. D 75, 035001 (2007); 76, 039902(E) (2007).

[50] F. Nagel, New aspects of gauge-boson couplings and the Higgs sector, Ph.D. thesis, Heidelberg University, 2004.

[51] M. Maniatis, A. von Manteuffel, O. Nachtmann, and F. Nagel, Eur. Phys. J. C 48, 805 (2006).

[52] A. Barroso, P. M. Ferreira, and R. Santos, Phys. Lett. B 632, 684 (2006).

[53] I. P. Ivanov, Phys. Rev. D 77, 015017 (2008).

[54] A. Barroso, P. M. Ferreira, I. P. Ivanov, and R. Santos, J. High Energy Phys. 06 (2013) 045.

[55] A. Barroso, P. M. Ferreira, I. P. Ivanov, R. Santos, and J. P. Silva, Eur. Phys. J. C 73, 2537 (2013).

[56] I. P. Ivanov, Phys. Rev. E 79, 021116 (2009).

[57] J. Horejsi and M. Kladiva, Eur. Phys. J. C 46, 81 (2006).

[58] S. Kanemura, T. Kubota, and E. Takasugi, Phys. Lett. B 313, 155 (1993).

[59] A. G. Akeroyd, A. Arhrib, and E.-M. Naimi, Phys. Lett. B 490, 119 (2000).

[60] F. Staub, Phys. Lett. B 776, 407 (2018).

[61] R. Coimbra, M. O. P. Sampaio, and R. Santos, Eur. Phys. J. C 73, 2428 (2013).

[62] R. Costa, R. Guedes, M. O. P. Sampaio, and R. Santos, SCANNERS project, 2014, http://scanners.hepforge.org.

[63] O. Deschamps, S. Monteil, V. Niess, S. Descotes-Genon, S. T'Jampens, and V. Tisserand, Phys. Rev. D 82, 073012 (2010).

[64] F. Mahmoudi and O. Stal, Phys. Rev. D 81, 035016 (2010).

[65] T. Hermann, M. Misiak, and M. Steinhauser, J. High Energy Phys. 11 (2012) 036.

[66] M. Misiak et al., Phys. Rev. Lett. 114, 221801 (2015).

[67] M. Misiak and M. Steinhauser, Eur. Phys. J. C 77, 201 (2017).

[68] G. Abbiendi et al. (LEP, DELPHI, OPAL, ALEPH, L3), Eur. Phys. J. C 73, 2463 (2013).

[69] H. E. Haber and H. E. Logan, Phys. Rev. D 62, 015011 (2000).

[70] M. Baak, J. Cúth, J. Haller, A. Hoecker, R. Kogler, K. Mönig, M. Schott, and J. Stelzer (Gfitter Group), Eur. Phys. J. C 74, 3046 (2014).

[71] G. Aad et al. (ATLAS, CMS), Phys. Rev. Lett. 114, 191803 (2015).

[72] P. Bechtle, O. Brein, S. Heinemeyer, G. Weiglein, and K. E. Williams, Comput. Phys. Commun. 181, 138 (2010).

[73] P. Bechtle, O. Brein, S. Heinemeyer, G. Weiglein, and K. E. Williams, Comput. Phys. Commun. 182, 2605 (2011).

[74] P. Bechtle, O. Brein, S. Heinemeyer, O. Stål, T. Stefaniak, G. Weiglein, and K. E. Williams, Eur. Phys. J. C 74, 2693 (2014).

[75] A. Djouadi, J. Kalinowski, and M. Spira, Comput. Phys. Commun. 108, 56 (1998).
[76] J. M. Butterworth et al., The Tools and Monte Carlo Working Group Summary Report from the Les Houches 2009 Workshop on TeV Colliders, in Physics at TeV colliders. Proceedings of 6th Workshop, dedicated to Thomas Binoth, Les Houches, France, June 8-26, 2009, 2010, arXiv:1003.1643.

[77] R. V. Harlander, S. Liebler, and H. Mantler, Comput. Phys. Commun. 184, 1605 (2013).

[78] R. V. Harlander, S. Liebler, and H. Mantler, Comput. Phys. Commun. 212, 239 (2017).

[79] G. Aad et al. ATLAS, CMS, J. High Energy Phys. 08 (2016) 045.

[80] P. M. Ferreira, R. Santos, M. Sher, and J. P. Silva, Phys. Rev. D 85, 077703 (2012).

[81] P. M. Ferreira, R. Guedes, M. O. P. Sampaio, and R. Santos, J. High Energy Phys. 12 (2014) 067.

[82] P. M. Ferreira, J. F. Gunion, H. E. Haber, and R. Santos, Phys. Rev. D 89, 115003 (2014).

[83] P. M. Ferreira, R. Santos, M. Sher, and J. P. Silva, Phys. Rev. D 85, 035020 (2012).

[84] R. Barate et al. (OPAL, DELPHI, LEP Working Group for Higgs boson searches, ALEPH, L3), Phys. Lett. B 565, 61 (2003).

[85] S. Schael et al. (DELPHI, OPAL, ALEPH, LEP Working Group for Higgs Boson Searches, L3), Eur. Phys. J. C 47, 547 (2006).

[86] R. D. Peccei and H. R. Quinn, Phys. Rev. Lett. 38, 1440 (1977).

[87] F. Staub, arXiv:0806.0538.

[88] F. Staub, Comput. Phys. Commun. 181, 1077 (2010).

[89] F. Staub, Comput. Phys. Commun. 182, 808 (2011).

[90] F. Staub, Comput. Phys. Commun. 185, 1773 (2014).

[91] F. Staub, Adv. High Energy Phys. 2015, 840780 (2015).

[92] J. Braathen, M. D. Goodsell, M. E. Krauss, T. Opferkuch, and F. Staub, Phys. Rev. D 97, 015011 (2018).

[93] S. P. Martin, Phys. Rev. D 91, 114003 (2015).

[94] S. P. Martin, Phys. Rev. D 92, 014026 (2015).

[95] S. P. Martin, Phys. Rev. D 67, 095012 (2003).

[96] B. Grinstein, C. W. Murphy, and P. Uttayarat, J. High Energy Phys. 06 (2016) 070.

[97] M. Krause, R. Lorenz, M. Muhlleitner, R. Santos, and H. Ziesche, J. High Energy Phys. 09 (2016) 143.

[98] M. Krause, M. Muhlleitner, R. Santos, and H. Ziesche, Phys. Rev. D 95, 075019 (2017).

[99] P. Basler, M. Muhlleitner, and J. Wittbrodt, J. High Energy Phys. 03 (2018) 061.

[100] P. Basler, M. Krause, M. Muhlleitner, J. Wittbrodt, and A. Wlotzka, J. High Energy Phys. 02 (2017) 121.

[101] P. M. Ferreira, I. Jack, and D. R. T. Jones, Phys. Lett. B 392, 376 (1997).

[102] J. Zinn-Justin, Phys. Rep. 70, 109 (1981).

[103] N. G. Deshpande and E. Ma, Phys. Rev. D 18, 2574 (1978).

[104] R. Barbieri, L. J. Hall, and V. S. Rychkov, Phys. Rev. D 74, 015007 (2006).

[105] L. L. Honorez, E. Nezri, J. F. Oliver, and M. H. Tytgat, J. Cosmol. Astropart. Phys. 02 (2007) 028.

[106] M. Krawczyk, D. Sokołowska, P. Swaczyna, and B. Świeżewska, J. High Energy Phys. 09 (2013) 055. 
[107] P. Draper, H. E. Haber, and J. T. Ruderman, J. High Energy Phys. 06 (2016) 124.

[108] G. C. Branco, P. M. Ferreira, L. Lavoura, M. N. Rebelo, M. Sher, and J. P. Silva, Phys. Rep. 516, 1 (2012).

[109] H. E. Haber and R. Hempfling, Phys. Rev. D 48, 4280 (1993).

[110] M. Sperling, D. Stöckinger, and A. Voigt, J. High Energy Phys. 07 (2013) 132.

[111] M. Sperling, D. Stöckinger, and A. Voigt, J. High Energy Phys. 01 (2014) 068.
[112] K. A. Olive et al. (Particle Data Group), Chin. Phys. C 38, 090001 (2014).

[113] A. Denner et al., Report No. Lhchxswg-int-2015-006, https://cds.cern.ch/record/2047636.

[114] L. H. C. S. Group, The LHC Higgs Cross Section Working Group Webpage at CERN, https://twiki.cern.ch/twiki/bin/ view/LHCPhysics/LHCHXSWG.

[115] S. Dittmaier et al. (LHC Higgs Cross Section Working Group), arXiv:1101.0593. 